\title{
A New Robust MRAC Using Variable Structure Design for Relative Degree Two Plants*†
}

\section{LI-CHEN FU}

\begin{abstract}
Variable structure design concept has been successfully incorporated to establish a new model reference adaptive controller for SISO plants with relative degree two with an aim to achieve good robustness, well-behaved transient responses, and good tracking performances.
\end{abstract}

Key Words-Model reference adaptive control; variable structure systems; robustness; global stability; output tracking.

\begin{abstract}
Variable structure design concept has been shown not only useful in dealing with uncertain systems but also useful in establishing controllers in the adaptive context. Continuing the work, this research proposed a new robust model reference adaptive control for single-input-singleoutput linear plants with relative degree two, a class which encompasses a large number of linearized mechanical systems. When compared with the conventional MRAC schemes, the current approach drastically improves the transient behavior and the tracking performance of the closed loop system from several numerical simulations. Furthermore, if the reference input satisfies the persistently exciting condition or if the prior knowledge about the interval in which the true parameter vector lies is available, the magnitude of the input force can be considerably reduced into a more realistic level. In the presence of mild unmodelled dynamics and bounded output disturbances, the proposed scheme possesses similar well behaved properties. It can be shown that the tracking error will, in fact, fall into a residual set whose size can be directly related to the size of unmodelled dynamics and of output disturbances.
\end{abstract}

\section{INTRODUCTION}

IN THE RECENT adaptive literature, a great deal of concentration is placed on the robustification of the adaptive control schemes ever since Rohrs et al. (1985) pointed out the extreme sensitivity of these schemes to assumptions such as known relative degree and freedom of disturbances. Unfortunately, instability has been observed and investigated, e.g. in Krause et al. (1983),

\footnotetext{
* Received 8 November 1989; revised 28 January 1991; received in final form 20 January 1992 . The original version of this paper was not presented at any IFAC meeting. This paper was recommended for publication in revised form by Associate Editor R. R. Bitmead under the direction of Editor P. C. Parks.

$\dagger$ The research is supported by National Science Council under the grant NSC79-0404-E-002-03.

‡Department of Electrical Engineering and Department of Computer Science \& Information Engineering, National Taiwan University, Taipei, Taiwan, R.O.C.
}

Ioannou and Kokotovic (1984a), Riedle and Kokotovic (1985), Åstrom (1985) and Fu and Sastry $(1987 \mathrm{a}, \mathrm{b})$ when some of the assumptions are violated. So far, these researches can be classified into three categories: (i) to ensure the condition of persistency of excitation (PE) in order to achieve the robustness of the controller; (ii) to modify the parameter adaptation law; (iii) to apply the variable structure design (VSD) concept. In the first category, a property of exponential stability of the nominal system is first guaranteed when the PE condition is satisfied and then either results with local stability are shown (e.g. in Kosut and Johnson, 1984; Bodson and Sastry, 1984; Chen and Cook, 1984; Bodson, 1986; Fu and Sastry, 1987b; Kokotovic et al., 1985) or those with global stability are obtained (e.g. in Kosut and Friedlander, 1985; Anderson et al., 1986). Several results have been obtained in the second category such as: concept of a dead zone is introduced in Egardt (1980), Peterson and Narendra (1982), Sastry (1984) and Kreisselmeier and Anderson (1986); restriction on the search region in the parameter space based on some prior bounds is given in the work in Egardt (1979) and Kreisselmeier and Narendra (1982); addition of a linear term $-\sigma \theta$ in the parameter update law, generally referred to as $\sigma$ modification, is adopted in Ioannou and Kokotovic (1984b), Ioannou (1986), Ioannou and Tsakalis (1986) and Ortega et al. (1987); and later $e_{1}$-modification is presented in Narendra and Annaswamy (1987) which differs from the aforementioned ones mainly in the additional term which is now replaced by $-\varepsilon_{1} \theta$. In the last one, the first attempt to apply VSD concept into 
model reference adaptive control (MRAC) scheme was made in Ambrosino et al. (1984) but the resulting controller is generally ill-posed; the so called VS-MRAC scheme (Hsu, 1990) incorporates switching on the adjustable parameters $\theta$ to achieve tracking performance; and the work in Fu (1991) applies switching not only to parameter adaptation but also to plant control input.

Continuing the work in Fu (1991), this paper presents a new robust continuous-time MRAC scheme for single-input-single-output (SISO) linear plants with relative degree two, a class which encompasses a huge number of linearized mechanical systems. Since the reference models for this class of plants fail to be strictly positive real (SPR), direct extension of the previous work to the present case is hardly achievable. Therefore, the proposed scheme incorporates the similar BSD concept in Fu (1991), but is based on the modified MRAC scheme proposed in Narendra and Valavani (1978) particularly for the case with relative degree two. Although this scheme shares the similarity with that in Ambrosino et al. (1984), the controller here is well-posed and the parameter update process, if not turned off, not only has contributed to the stabilization of the overall system but also has shared the load upon the plant input in handling the parameter uncertainties. The latter turns out to reduce the magnitude of the input force considerably. However, if some prior knowledge of the tight intervals in which the system parameters lie is available, then the update process can, in fact, be turned off so as to lower down the input level even more. On the other hand, the main difference of our scheme from the VS-MRAC scheme introduced in Hsu (1990) is that there they applied the VSD concept successively to the parameter adaptation, namely, parameter switching, to handle the parameter uncertainties whereas here that concept is mainly applied to the input synthesis. Further, the effect of replacing the so called equivalent control by the so called average control in the general VS-MRAC scheme may require further assessment in terms of stability and convergence properties. As opposed to this, the present scheme has taken into account the implementation issues and has been well investigated with complete analysis.

The striking features of the proposed MRAC scheme in this paper are the drastic improvement of both transient behavior and tracking performance from the conventional schemes (Narendra and Valavani, 1978; Narendra et al., 1980; Ioannou and Tsakalis, 1986) which are clearly demonstrated in several numerical simulations. Moreover, the magnitude of the input force here in general can be reduced considerably into a more realistic level, which allows one to avoid the occasion with input saturation. Besides, it is worth mentioning that the scheme is robust to unmodelled dynamics and bounded output disturbance, and the tracking accuracy can be directly related to the size of the non-ideal factors. Because of these properties, such a robust control has a potential to become a pragmatically feasible MRAC scheme.

The paper is organized as follows: in Section 2 , we formulate the model reference adaptive control problem for continuous-time, SISO linear plants with relative degree two; Section 3 describes the structure of the adaptive controller; investigation of the proposed MRAC scheme in the absence of unmodelled dynamics and output disturbances but in face of various situations is given in Section 4; in Section 5, robustness of the proposed scheme is analyzed in the presence of unmodelled dynamics and bounded output disturbance, and the tracking accuracy is related to the degree of those non-ideality; some numerical simulation examples are provided in Section 6 to demonstrate the effectiveness of the proposed MRAC scheme; finally, some conclusions are drawn in Section 7 .

\section{PROBLEM FORMULATION}

The problem to be treated in this paper is similar to the one in Fu (1991) but with relative degree two. In addition, some slightly different assumptions on the plant are made to pose the problem properly. It is now stated in the following:

Consider an SISO, linear time-invariant plant described by the following transfer function:

$$
\begin{aligned}
\hat{P}_{u}(s) & =\hat{P}(s)\left(1+\mu \Delta \hat{P}_{1}(s)\right)+\mu \Delta \hat{P}_{2}(s), \\
& =k_{p} \frac{\hat{n}_{p}(s)}{\hat{d}_{p}(s)}\left(1+\mu \Delta \hat{P}_{1}(s)\right)+\mu \Delta \hat{P}_{2}(s),
\end{aligned}
$$

where $\hat{P}(s)$ represents the nominal plant transfer function of order $n$, and $\mu \Delta \hat{P}_{1}(s)$ and $\mu \Delta \hat{P}_{2}(s)$ are the multiplicative and additive unmodelled dynamics, respectively with some $\mu \in R$ (Ioannou, 1986; Narendra and Annaswamy, 1987), satisfying the following.

\section{Assumptions.}

(A1) $\hat{n}_{p}(s)$ and $\hat{d}_{p}(s)$ are monic coprime polynomials of known degrees $n-2$ and $n$, respectively.

(A2) The sign of $k_{p}$ is known, and we assume it is positive without loss of generality. 
(A3) The nominal plant transfer function $\hat{P}(s)$ is minimum phase, i.e. $\hat{n}_{p}(s)$ is a Hurwitz polynomial.

(A4) $\Delta \hat{P}_{1}(s)$ and $\hat{P}^{-1} \Delta \hat{P}_{2}(s)$ are both stable proper transfer functions. Furthermore, suppose that the plant is operated subject to a bounded piecewise differentiable output disturbance $\xi_{0}$, i.e.

$$
\hat{y}_{p}=y_{p}+\zeta_{0}
$$

where $\left|\zeta_{0}\right| \leq \rho$ and, for almost everywhere in $t,\left|\dot{\zeta}_{0}\right| \leq \rho_{d}$ for some $\rho, \rho_{d}>0$.

The reference model is then described by the following transfer function:

$$
\hat{M}(s)=k_{m} \frac{\hat{n}_{m}(s)}{\hat{d}_{m}(s)},
$$

where $\hat{n}_{m}(s)$ and $\hat{d}_{m}(s)$ are monic but not necessarily coprime polynomials of degree $n-2$ and $n$, respectively. The model transfer function satisfies the following.

\section{Assumptions.}

(A5) $\hat{M}(s)$ is chosen to be stable and minimum phase. In addition, there exists $\hat{L}(s)=$ $(s+a)$ for some $a>0$ such that $\hat{M}(s) \hat{L}(s)$ is strictly positive real (SPR) (Anderson $e t$ al., 1986; Annaswamy and Narendra, 1988).

(A6) The sign of $k_{m}$ is the same as that of $k_{p}$, i.e. $k_{m}>0$.

Now the control objective can be stated as follows: under the condition that all the coefficients of the plant transfer function $\hat{P}_{u}(s)$ are not known precisely but subject to Assumptions (A1)-(A4), choose a suitable adaptive control law so that, for arbitrary bounded reference input $r(t)$, the overall system is stabilized despite the existence of unmodelled dynamics and bounded output disturbance. Furthermore, the plant output $y_{p}$ will try to follow the model output $y_{m}$ as closely as possible.

Here, the basic controller structure is chosen to be the one in Narendra and Valavani (1978), corresponding to the case of relative degree two. As shown in Fig. 1, the dynamical compensator blocks $F_{1}$ and $F_{2}$ are identical single-input, (n-1)-output systems described by transfer functions:

$$
\hat{F}_{1}(s)=\hat{F}_{2}(s)=\frac{1}{\hat{\Lambda}(s)}\left(\begin{array}{c}
1 \\
s \\
\vdots \\
s^{n-2}
\end{array}\right) \in R^{n-1}(s) .
$$

The monic characteristic polynomial $\hat{\Lambda}(s)$ is chosen such that $\hat{\Lambda}(s)=\hat{n}_{m}(s) \hat{L}(s)$ where $\hat{L}(s)$ is

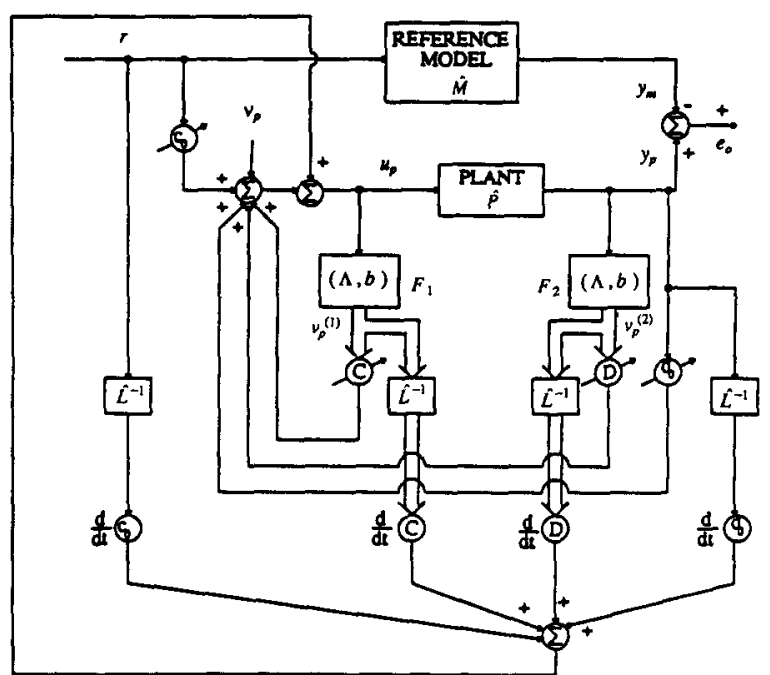

FIG. 1. Structure of model reference adaptive controller.

the one in Assumption (A5). There are a total of $2 n$ parameters to be tuned for the controller due to lack of precise knowledge of $\hat{n}_{p}(s)$ and $\hat{d}_{p}(s)$. The parameters $C \in R^{n-1}$ in the precompensator block serve to locate the closed loop plant zeros, while $D \in R^{n-1}$ and $d_{0} \in R$ in the postcompensator block assign the closed loop plant poles. The parameter $c_{0} \in R$ then adjusts the overall gain of the closed loop plant. The parameter vector $\theta$ is thus defined as:

$$
\theta^{T}=\left[c_{0}, C^{T}, d_{0}, D^{T}\right]^{T} .
$$

Refer to the previous problem statement, it can now be restated as how to synthesize the control input $u_{p}$ and on-line update the parameter vector $\theta$ so that the aforementioned objective can be met. In other words, a suitable control law and a parameter adaptation law will have to be devised to achieve the purpose. These will be investigated in the following sections.

\section{PRELIMINARIES}

The control scheme to be presented here is an outgrowth of the one in Fu (1991) which only deals with SISO plants with relative degree one. Define the signal vectors $w$ and $\hat{w}: R_{+} \rightarrow R^{2 n}$ as:

$$
\hat{w}=\left(\begin{array}{c}
r \\
v_{p}^{(1)} \\
\hat{y}_{p} \\
v_{p}^{(2)}
\end{array}\right)=\left(\begin{array}{c}
r \\
v_{p}^{(1)} \\
y_{p}+\zeta_{0} \\
v_{p}^{(2)}
\end{array}\right)=w+\left(\begin{array}{l}
0 \\
0 \\
\zeta_{0} \\
0
\end{array}\right)
$$

Note that for any differentiable function $h_{1}(\cdot): R_{+} \rightarrow R$ and measurable function $h_{2}(\cdot): R_{+} \rightarrow R$,

$$
\begin{aligned}
& \hat{L}(s)\left(h_{1}(t) \hat{L}^{-1}(s)\left(h_{2}\right)(t)\right) \\
& =h_{1}(t) h_{2}(t)+\frac{\mathrm{d}}{\mathrm{d} t} h_{1}(t) \hat{L}^{-1}(s)\left(h_{2}\right)(t),
\end{aligned}
$$


where $\hat{L}(s)$ is as given before and we use $\hat{L}^{-1}(s)\left(h_{2}\right)(t)$ to denote the filtered version of $h_{2}(t)$ with filter $\hat{L}^{-1}(s)$. Define the plant input $u_{p}$ as:

$$
u_{p}=\left(\theta^{T} \hat{w}+\dot{\theta}^{T} \hat{L}^{-1}(s)(\hat{w})\right)+v_{p},
$$

which is an implementable input signal once $\dot{\theta}$ (gives a suitable parameter adaptation law) and $v_{p}$ are specified and from equation (7) is, in fact, equivalent to the following:

$$
u_{p}=\hat{L}(s)\left(\theta^{T} \hat{L}^{-1}(s)(\hat{w})\right)+v_{p} .
$$

Note that $v_{p}$ is an additional input to be given later. It can be shown from Narendra and Valavani (1978) that there exist unique constant parameters $\theta^{*} \in R^{2 n}$ such that, in the absence of $\Delta \hat{P}_{1}(s), \Delta \hat{P}_{2}(s), \zeta_{0}$, and with $v_{p}=0$ in equations (8) and (9), the transfer function of the plant plus the controller equals that of the model, $\hat{M}(s)$, when $\theta \equiv \theta^{*}$. This result also directly follows from Narendra and Valavani (1978) since

$$
\hat{L}(s)\left(\theta^{* T} \hat{L}^{-1}(s)(\hat{w})\right) \equiv \theta^{* T} \hat{w} .
$$

This, in turn, implies that in the ideal case with $v_{p}=0$

$$
y_{p}=\hat{P}(s)\left(\theta^{* T} w\right)=\hat{M}(s)(r)=y_{m} .
$$

Now let $\phi=: \theta-\theta^{*}$ denote the parameter error vector, and $\xi=: \hat{L}^{-1}(s)(\hat{w})$ and $\xi=$ : $\hat{L}^{-1}(s)(w)$ denote the filtered version of $\hat{w}$ and $w$, respectively through $\hat{L}^{-1}(s)$. Then, it can be verified that with the choice of $u_{p}$ in equation (9),

$$
\begin{aligned}
y_{p}= & \hat{P}(s)\left(1+\mu \Delta \hat{P}_{1}(s)\right)\left(u_{p}\right)+\mu \Delta \hat{P}_{2}(s)\left(u_{p}\right) \\
= & \hat{P}(s)\left(\theta^{* T} \hat{w}\right)+\hat{P}(s)\left(\hat{L}(s)\left(\phi^{T \xi}\right)+v_{p}\right) \\
& +\mu\left(\hat{P}(s) \Delta \hat{P}_{1}(s)+\Delta \hat{P}_{2}(s)\right)\left(u_{p}\right) \\
= & y_{m}+\frac{1}{c_{0}^{*}} \hat{M}(s)\left(\hat{L}(s)\left(\phi^{T} \xi\right)+v_{p}\right) \\
& +\frac{1}{c_{0}^{*}} \hat{M}(S)\left(D^{* T} \hat{F}_{2}(s)+d_{0}^{*}\right)\left(\zeta_{0}\right) \\
& +\mu\left(1+\frac{1}{c_{0}^{*}} \hat{M}(s)\left(D^{* T} \hat{F}_{2}(s)+d_{0}^{*}\right)\right) \\
& \times\left(\hat{P}(s) \Delta \hat{P}_{1}(s)+\Delta \hat{P}_{2}(s)\right)\left(u_{p}\right) .
\end{aligned}
$$

Denote $e_{0}=y_{p}-y_{m}$, then equation (12) can be written as:

$$
e_{0}=\frac{1}{c_{0}^{*}} \hat{M}(s) \hat{L}(s)\left(\phi^{T} \hat{\xi}+\hat{L}^{-1}(s)\left(v_{p}\right)+\eta_{0}+\mu \eta_{i}\right),
$$

where

$$
\eta_{0}=\hat{L}^{-1}(s)\left(D^{* T} \hat{F}_{2}(s)+d_{0}^{*}\right)\left(\zeta_{0}\right)=\hat{\Delta}_{\eta_{0}}(s)\left(\zeta_{0}\right)
$$

and

$$
\begin{aligned}
\eta_{i}= & \left(\hat{M}^{-1}(s)+\frac{1}{c_{0}^{*}}\left(D^{* T} \hat{F}_{2}(s)+d_{0}^{*}\right)\right) \\
& \times\left(\hat{P}(s) \Delta \hat{P}_{1}(s)+\Delta \hat{P}_{2}(s)\right) \hat{L}^{-1}(s)\left(u_{p}\right) \\
= & \hat{\Delta}_{\eta_{i}}(s) \hat{L}^{-1}(s)\left(u_{p}\right) .
\end{aligned}
$$

Here, it can be easily verified that $\hat{\Delta}_{\eta_{i}}(s)$ and $\hat{\Delta}_{\eta_{0}}(s)$ are stable proper and strictly proper transfer functions, respectively. Since we have assumed $\left|\zeta_{0}\right| \leq \rho$, equation (14) will imply that $\left|\eta_{0}\right| \leq b_{1} \rho$ for some $b_{\rho}>0$. Note that from Assumptions (A4)-(A5), a state space representation of the error system given by equations (13) $-(15)$ can be readily obtained as:

$$
\begin{aligned}
\dot{e} & =A_{m} e+b_{m}\left(\phi^{T} \hat{\xi}+\hat{L}^{-1}(s)\left(v_{p}\right)+\eta_{0}+\mu \eta_{i}\right), \\
e_{0} & =c_{m}^{T} e
\end{aligned}
$$

and

$$
\begin{aligned}
\dot{x}_{\zeta} & =A_{\zeta} x_{\zeta}+b_{\zeta} \hat{L}^{-1}\left(u_{p}\right), \\
\eta_{i} & =c_{\zeta}^{T} x_{\zeta}+d_{\zeta} \hat{L}^{-1}\left(u_{p}\right),
\end{aligned}
$$

where $\left(A_{m}, b_{m}, c_{m}\right)$ and $\left(A_{\zeta}, b_{\zeta}, c_{\zeta}, d_{\zeta}\right)$ are minimal realizations of $\frac{1}{c_{0}^{*}} \hat{M}(s) \hat{L}(s)$ and $\hat{\Delta}_{\eta_{t}}(s)$, respectively. In particular, $A_{m}$ and $A_{\zeta}$ are all Hurwitz matrices.

\section{ADAPTIVE CONTROLLER DESIGN}

In the previous section, in order to realize the input signal $u_{p}$ in equation (8), the time derivative of $\theta$, namely, $\dot{\theta}$, and the additional signal $v_{p}$ have to be specified. The former is given by the parameter adaptation law whereas the latter is given by the control law.

Define $\hat{e}_{0}=e_{0}+\xi_{0}=\hat{y}_{p}-y_{m}$ and consider the modified gradient parameter adaptation law:

$$
\begin{gathered}
\dot{\theta}=\dot{\phi}=-\Gamma\left(\hat{e}_{0} \xi+\sigma \theta\right), \\
\sigma= \begin{cases}0 & \text { if }|\theta|<\theta_{0} \\
\sigma_{0}\left(\frac{|\theta|}{\theta_{0}}-1\right)\left(1+|\xi|^{2}\right) & \text { if } \theta_{0} \leq|\theta|<2 \theta_{0} \\
\sigma_{0}\left(1+|\xi|^{2}\right) & \text { if }|\theta| \geq 2 \theta_{0},\end{cases}
\end{gathered}
$$

where $|\cdot|$ denotes the Euclidian vector norm, $\Gamma(>0)$ is the adaptation gain matrix, and $\sigma_{0}(>0)$, $\theta_{0}\left(\geq\left|\theta^{*}\right|\right)$ are some design parameters. Such an adaptation law is reminiscent of the one in Ioannou and Tsakalis (1986).

Given equations (8) and (18), in the rest of this section, focus will be placed on the choice of $v_{p}$ so that the overall system is guaranteed to be globally stable and the tracking error is ensured to converge to a residual set whose size is a simple function of $\mu$ and $\rho$ while they are small. Let $v_{p}$ be constructed as follows: for some 
sufficiently small $\Delta t(>0)$ and $\varepsilon(>0)$,

$$
\begin{aligned}
& v_{p}=a f\left(\hat{e}_{0}\right) \pi(|\xi|)+f\left(\hat{e}_{0}\right) \frac{\mathrm{d}}{\mathrm{d} t} \pi(|\xi|) \\
& +\frac{1}{\Delta t}\left(f\left(\hat{e}_{0}(t)\right)-f\left(\hat{e}_{0}(t-\Delta t)\right)\right) \pi(|\xi|),
\end{aligned}
$$

where

$$
f\left(\hat{e}_{0}\right)= \begin{cases}-\operatorname{sgn}\left(\hat{e}_{0}\right) & \left|\hat{e}_{0}\right| \geq \varepsilon \\ -\frac{\hat{e}_{0}}{\varepsilon} & \left|\hat{e}_{0}\right|<\varepsilon\end{cases}
$$

with

$$
\operatorname{sgn}(x)=\left\{\begin{array}{rl}
+1 & x>0 \\
0 & x=0 \\
-1 & x<0
\end{array}\right.
$$

and $\pi(\cdot): R_{+} \rightarrow R_{+}$is some properly chosen differentiable function.

\section{Remarks.}

(1) The function $f\left(\hat{e}_{0}\right)$ given in equation (20) in general is not a differentiable function. However, if we can show that $f\left(\hat{e}_{0}\right)$, is, in fact, a piecewise differentiable function, then the signal $v_{p}$ in equation (19) can be re-expressed as:

$$
\hat{L}^{-1}(s)\left(v_{p}\right)=f\left(\hat{e}_{0}\right) \pi(|\xi|)+\Delta\left(\hat{e}_{0}\right),
$$

where $\Delta\left(\hat{e}_{0}\right)$ satisfies $(\mathrm{Fu}, 1992)$

$\left|\Delta\left(\hat{e}_{0}\right)\right| \leq \frac{\Delta t}{\varepsilon}\left(\left\|\dot{e}_{0 t}\right\|_{\infty}+\rho_{d}\right)\left(2\left\|\pi_{t}\right\|_{\infty}+\frac{1}{a}\left\|\dot{\pi}_{t}\right\|_{\infty}+\gamma_{1}\right)$,

whenever $\max \left(\left|\hat{e}_{0}(t)\right|,\left|\hat{e}_{0}(t-\Delta t)\right|\right)<\varepsilon$ for some $\gamma_{1} \geq 0$, whereas $\Delta\left(\hat{e}_{0}\right)=\varepsilon^{\prime}(t)$ with $\varepsilon^{\prime}(t)$ denoting an exponentially decaying signal (we hereafter abuse the notation $\varepsilon^{\prime}(t)$ to denote all the suitably defined exponentially decaying signals) whenever $\min \left(\left|\hat{e}_{0}(t)\right|,\left|\hat{e}_{0}(t-\Delta t)\right|\right)>\varepsilon$. Suppose that all the signals inside the closed loop system are uniformly bounded, then, it is easily seen that there exists $\bar{\delta}>0$ such that $\left|\Delta\left(\hat{e}_{0}(t)\right)\right| \leq \bar{\delta} \Delta t$ for all $t \geq 0$ when $\Delta t$ is small. Thus, in general the smaller $\Delta t$ is, the smaller $\Delta\left(\hat{e}_{0}\right)$ will be. Now with equation (22), the error system in equation (16) can be more concisely expressed as:

$$
\begin{aligned}
\dot{e}= & A_{m} e+b_{m}\left(\phi^{T} \xi+f\left(\hat{e}_{0}\right) \pi(|\xi|)\right. \\
& \left.+\Delta\left(\hat{e}_{0}\right)+\eta_{0}+\mu \eta_{i}\right), \\
e_{0}= & c_{m}^{T} e .
\end{aligned}
$$

(2) Seemingly, the implementation of $v_{p}$ in equation (19) will require differentiation of the signal $\pi(\cdot)$. However, later in the sequel we will explicitly specify the function $\pi(\cdot)$ (and, in turn, $\left.\frac{\mathrm{d}}{\mathrm{d} t} \pi(\cdot)\right)$ so that no explicit differentiation is ever required.

This adaptive controller will first be investigated in the ideal case where $\Delta \hat{P}_{1}(s)=$ $\Delta \hat{P}_{2}(s)=\zeta_{0} \equiv 0$. The general (non-ideal) case will be treated in Section 5. Therefore, $\hat{e}_{0}=e_{0}$ and $\xi=\xi$ so that the equation (16) can be concisely rewritten as:

$$
\begin{aligned}
\dot{e} & =A_{m} e+b_{m}\left(\phi^{T \xi}+\hat{L}^{-1}(s)\left(v_{p}\right)\right), \\
e_{0} & =c_{m}^{T} e .
\end{aligned}
$$

To study the stability property of the overall adaptive system, Lyapunov analysis is adopted here, i.e. to construct a function $V(e, \phi)$ as follows:

$$
V(e, \phi)=\frac{1}{2} e^{T} P_{e} e+\frac{1}{2} \phi^{t} \Gamma^{-1} \phi
$$

and to evaluate its time derivative:

$$
\begin{aligned}
\dot{V}(e, \phi)= & \frac{1}{2} e^{T}\left(A_{m}^{T} P_{e}+P_{e} A_{m}\right) e \\
& +e^{T} P_{e} b_{m}\left(\phi^{T} \xi+\hat{L}^{-1}(s)\left(v_{p}\right)\right) \\
& -e_{0} \phi^{T} \xi-\sigma \phi^{T} \theta,
\end{aligned}
$$

where $P_{e}(>0)$ satisfies the following properties:

$$
-\left(A_{m}^{T} P_{e}+P_{e} A_{m}\right)=2 Q_{e}(>0) \text { and } P_{e} b_{m}=c_{m} \text {, }
$$

due to the SPR property of the transfer function $\hat{M}(s) \hat{L}(s)$ in Assumption (A5). In particular, there exist $p_{e 1}, p_{e 2}>0$ and $q_{e 1}, q_{e 2}>0$ such that $p_{e 1} I \leq P_{e} \leq p_{e 2} I$ and $q_{e 1} I \leq Q_{e} \leq q_{e 2} I$.

Now we are ready to state our main results on the stability as well as the tracking properties of the ideal adaptive system in the following theorem.

Theorem 1. (Global stability and output tracking.) Consider the previous problem set-up and choose $\pi(|\xi|)$ as follows:

$$
\pi(|\xi|)= \begin{cases}\beta_{1}|\xi|+\beta_{2} & |\xi| \geq h \\ \beta\left(\frac{|\xi|^{2}}{2 h}+\frac{h}{2}\right)+\beta_{2}, & |\xi|<h\end{cases}
$$

where $\beta_{1} \geq 0, \beta_{2}>0$, and $h$ is a very small positive number. Then all signals inside the closed loop system are uniformly bounded, i.e. $e(\cdot) \in L_{\infty}^{n}, w(\cdot) \in L_{\infty}^{2 n}$, and $\theta(\cdot) \in L_{\infty}^{2 n}$, and the size of the residual set of $e_{0}$ is a class $K$ function of $\varepsilon$ provided that $\Delta t$ is sufficiently small. Furthermore, if $\beta_{1}$ and $\beta_{2}$ are chosen large enough, then the residual set is exactly the interval $[-\varepsilon, \varepsilon]$, and the set-convergence rate is at least linear in time.

Remark. It can be verified that $\pi(|\xi|)$ given in equation (29) is indeed a differentiable function 
and $\frac{\mathrm{d}}{\mathrm{d} t} \pi(\cdot)$ is actually implemented as:

$$
\frac{\mathrm{d}}{\mathrm{d} t} \pi(|\xi|)= \begin{cases}-\beta_{1} \frac{\xi^{T}}{|\xi|}(a \xi-w) & |\xi| \geq h \\ -\beta_{1} \frac{\xi^{T}}{h}(a \xi-w) & |\xi|<h .\end{cases}
$$

Furthermore, $\pi(|\xi|)$ satisfies

$$
\beta_{1}|\xi|+\beta_{2} \leq \pi(|\xi|) \leq \beta_{1}|\xi|+\beta_{2}^{\prime},
$$

where $\beta_{2}^{\prime}=\left(\frac{\beta_{1}}{2} h+\beta_{2}\right)$.

Proof of Theorem 4.1. First, we will show that all signals inside the closed loop system belong to the extended $L_{\infty}$ space (Vidyasagar, 1978), i.e. $e(\cdot) \in L_{\infty e}^{n}, \quad w(\cdot) \in L_{\infty e}^{2 n}$, and $\theta(\cdot) \in L_{\infty e}^{2 n}$ by investigating the time derivative of the function $V(e, \phi)$. This investigation will proceed via a contradiction argument, i.e. there exists $T>0$ such that some signal inside the system becomes unbounded at $t=T$. But this presumption implies that all signals remain bounded for all $t \in[0, T)$ so that from equations (20) and (25) $f\left(e_{0}(t)\right)$ is indeed a piecewise differentiable function for all $t \in[0, T)$. From the previous remark (1) in the beginning of this section, we can readily conclude that the system given in equation (25) is actually equivalent to the following system over that time interval.

$$
\begin{aligned}
\dot{e} & =A_{m} e+b_{m}\left(\phi^{T} \xi+f\left(e_{0}\right) \pi(|\xi|)+\Delta\left(e_{0}\right)\right), \\
e_{0} & =c_{m}^{T} e .
\end{aligned}
$$

As a consequence, equation (27) is simplified as:

$$
\begin{aligned}
\dot{V}(e, \phi)= & -e^{T} Q_{e} e-\sigma \phi^{T} \theta+e_{0} f\left(e_{0}\right) \pi(|\xi|) \\
& +e_{0} \Delta\left(e_{0}\right) .
\end{aligned}
$$

Due to the fact that $\sigma \phi^{T} \theta \geq 0$ and the previous remark about $\Delta\left(e_{0}\right)$, whenever $e_{0}$ is bounded away from the interval $[-\varepsilon, \varepsilon]$, equation (33) becomes

$$
\dot{V}(e, \phi) \leqslant-q_{e 1}|e|^{2}-\left|e_{0}\right|\left(\beta_{2}-\varepsilon^{\prime}(t)\right) .
$$

Since $\varepsilon^{\prime}(t)$ decays exponentially in $t, \dot{V}$ in equation (34) will become negative after some time elapses. This implies that $e_{0}$ cannot grow unbounded at $t=T$ and, hence, neither can the parameter error vector $\phi(t)$ and the regressor vector $w(t)$ due to Lemma $\mathrm{A} 1$ and Lemma $\mathrm{A} 2$, respectively (see Appendix for Lemmas and their proofs). This, of course, also implies that $\xi(t)$ remains bounded on $[0, T]$. Therefore, by contradiction, all signals inside the closed loop belong to the extended $L_{\infty}$ space so that equation (33) remains valid for all $t \geq 0$. This further implies that there exist $\Delta t_{1}>0$ such that
$\left|\Delta\left(e_{0}\right)\right| \leq \delta(t) \Delta t$ for all $\Delta t \in\left[0, \Delta t_{1}\right]$ and for some $\delta(\cdot) \in L_{\infty e}$ because of equations (23), (30), (32), Lemma A3, and the small gain theorem (Desoer and Vidyasagar, 1975). However, $\delta(t) \leq \bar{\delta}$ for all $t \geq 0$ if $e(t), w(t)$, and $\phi(t)$ are all uniformly bounded.

Now, since equation (33) is valid for all $t \geq 0$, it is clear that

$$
\dot{V}(e, \phi) \leq-q_{e 1}|e|\left(|e|-\Delta t\left(\frac{\left|c_{m}\right| \delta(t)}{q_{e 1}}\right)\right),
$$

which according to Lemma A1 and Lemma A3 shows that if $\left\|e_{t}\right\|_{\infty}<l_{1}(>0)$, then there exist $l_{2}(\cdot), \quad l_{3}(\cdot)>0$ such that $\left\|\delta_{t}\right\|_{\infty}<l_{2}\left(l_{1}\right)$ and $\left\|V_{t}\right\|_{\infty}<l_{3}\left(l_{1}\right)$. On the other hand, the latter inequality, in turn, implies that $\left\|e_{t}\right\|_{\infty} \leq$ $\sqrt{\frac{2 l_{3}\left(l_{1}\right)}{p_{e 1}}}$. Combining these facts along with equation (35), we can easily see that if we choose $\Delta t_{2}$ to be $\min \left(\Delta t_{1}, l_{0}\left(\frac{q_{e 1}}{\left|c_{m}\right|} \frac{l_{1}}{l_{2}}\right)\right)$ where $l_{0}=\frac{|e(0)|}{l_{1}}$, then there exists sufficiently large $l_{1}$ so that, for all $t \geq 0, V(t) \leq l_{3}\left(l_{0} l_{1}\right) \leq l_{3}\left(l_{1}\right)$ and, hence, $\left\|e_{t}\right\|_{\infty} \leq \sqrt{\frac{2 l_{3}\left(l_{0} l_{1}\right)}{p_{e 1}}}<l_{1}$. Consequently, $e(\cdot) \in L_{\infty}^{n}$ and $\phi(\cdot) \in L_{\infty}^{2 n}$, which, in turn, implies that $\xi(\cdot) \in L_{\infty}^{2 n}$ as a result of Lemma A3. From the proof of Lemma $A 2$, it can be verified that $w(\cdot) \in L_{\infty}^{2 n}$ as well.

To show the size of the residual set is a class $K$ function of $\varepsilon$, further investigation of equation (34) has to be taken. Assume that initially $e_{0}$ stays outside the interval $[-\varepsilon, \varepsilon]$. Then $|e| \geq$ $\frac{\left|e_{0}\right|}{\left|c_{m}\right|} \geq \frac{\varepsilon}{\left|c_{m}\right|}$ and hence, after some time $t_{1}>0$ equation (34) can be rewritten as:

$$
\begin{aligned}
\dot{V}(e, \phi) & \leq-\frac{q_{e 1}}{\left|c_{m}\right|^{2}}\left|e_{0}\right|^{2}-\left|e_{0}\right|\left(\beta_{2}-\varepsilon^{\prime}(t)\right) \\
& \leq-\left(\frac{q_{e 1}}{\left|c_{m}\right|^{2}} \varepsilon^{2}+\frac{1}{2} \beta_{2} \varepsilon\right) \quad t \geq t_{1},
\end{aligned}
$$

which implies that $V$ is strictly decreasing during this period so that there exists $t_{2} \geq t_{1}$ such that $\left|e_{0}\left(t_{2}\right)\right| \leq \varepsilon$. However, once $e_{0}$ falls into the interval $[-\varepsilon, \varepsilon]$, equation (36) is no longer valid and, hence, $V$ may be increasing so that $e_{0}$ eventually leaves that interval again. To find out the level of magnitude up to which $\left|e_{0}\right|$ will grow, we augment equation (36) via the use of equation (33) so that

$$
\dot{V}(e, \phi) \leq-\left(\frac{q_{e 1}}{\left|c_{m}\right|^{2}} \varepsilon^{2}+\beta_{2} \varepsilon\right)+\sqrt{\frac{2}{p_{e 1}}}\left|c_{m}\right| \bar{\delta} \Delta t V^{1 / 2},
$$


which readily leads to the following conclusion:

$$
\left|e_{0}\right| \leq \sqrt{\frac{2}{p_{e 1}}}\left|c_{m}\right| V^{1 / 2} \leq \frac{1}{\bar{\delta} \Delta t}\left(\frac{q_{e 1}}{\left|c_{m}\right|^{2}} \varepsilon^{2}+\beta_{2} \varepsilon\right),
$$

and, hence, the size of the residual set of $e_{0}$ is indeed a class $K$ function of $\varepsilon$. So far, we have concluded the first part of the proof.

In order to show that the residual set is exactly $[-\varepsilon, \varepsilon]$ when $\beta_{1}$ and $\beta_{2}$ are chosen properly large, we differentiate the output equation (32) so that

$$
\begin{aligned}
e_{0} \cdot \dot{e}_{0}= & e_{0}\left(c_{m}^{T} A_{m} e+c_{m}^{T} b_{m}\left(\phi^{T} \xi\right.\right. \\
& \left.\left.+f\left(e_{0}\right) \pi+\Delta\left(e_{0}\right)\right)\right),
\end{aligned}
$$

which, whenever $\left|e_{0}\right|>\varepsilon$, provides an estimate of the rate of change of $\left|e_{0}\right|$ as:

$$
\begin{aligned}
\frac{1}{2} \frac{\mathrm{d}}{\mathrm{d} t}\left|e_{0}\right|^{2} \leq- & \left|e_{0}\right|\left(k_{p}\left(\beta_{2}-\frac{1}{k_{p}} \| c_{m}^{T} A_{m}|| e \mid-\bar{\delta} \Delta t\right)\right. \\
& \left.+k_{p}\left(\beta_{1}-|\phi|\right)|\xi|\right)
\end{aligned}
$$

where the fact $c_{m}^{T} b_{m}=k_{p}$ is used. Since $\phi$ and $e$ are uniformly bounded, so long as $\beta_{1}$ and $\beta_{2}$ can be chosen such that $\beta_{1} \geq \sup _{t \geq t_{f_{c}}}|\phi(t)|$ and $\beta_{2}>\sup _{t \geq t_{f_{c}}}$ $\frac{1}{k_{p}}\left\|c_{m}^{T} A_{m}\right\||e(t)|+\bar{\delta} \Delta t$ for some finite $t_{f_{c}}>0$, equation (40) can be shown to satisfy

$$
\frac{1}{2} \frac{\mathrm{d}}{\mathrm{d} t}\left|e_{0}\right|^{2} \leq-\beta_{3}\left|e_{0}\right|
$$

for some $\beta_{3}>0$ or, equivalently,

$$
\frac{\mathrm{d}}{\mathrm{d} t}\left|e_{0}\right| \leq-2 \beta_{3},
$$

for all $t \geq t_{f c}$. This shows that $e_{0}$ will fall into the interval $[-\varepsilon, \varepsilon]$ at least at the linear rate and will never leave that interval afterwards. This completes the second part of the proof. Q.E.D.

\section{Remarks.}

(1) In the theorem, when $\beta_{1}$ and $\beta_{2}$ are chosen properly large, $e_{0}$ will actually fall into the interval $[-\varepsilon-\Delta \varepsilon, \varepsilon+\Delta \varepsilon]$ for some small $\Delta \varepsilon>0$ because the statement $\left|e_{0}\right|>\varepsilon$ should, in fact, be replaced by $\min \left(\left|e_{0}(t)\right|,\left|e_{0}(t-\Delta t)\right|\right)>\varepsilon$. Since $\Delta t$ is usually very small, $\Delta \varepsilon$ is also kept extremely small. Therefore, in the following we will not differentiate the two testing conditions and will neglect $\Delta \varepsilon$ simply for ease of presentation.

(2) Suppose $\varepsilon$ is very small and $\beta_{1}, \beta_{2}$ are chosen sufficiently large as required in the proof, then the theorem is obviously a drastic improvement of the transient response as well as the tracking performance of the MRAS from those conventional schemes (Narendra and Valavani, 1978; Narendra et al., 1980; Ioannou and Tsakalis, 1986).

(3) In order to guarantee that $\beta_{1}$ and $\beta_{2}$ are chosen indeed properly large but under the situation that $\theta^{*}$ is not known and the state $e$ is not available, some conservative estimates have to be used. From equation (38), $V^{1 / 2}$ and, hence, $|\phi|$ are class $K$ functions of $\varepsilon$ after some time. If $\varepsilon$ is sufficiently small, $\beta_{1}$ only needs to be chosen to satisfy $\beta_{1} \geq \theta_{0}$ (generally $\gg \varepsilon$ ) and the bound on $|e|$ can be obtained through an estimation from equations (19) and (25). However, the magnitude of the resulting control input may, thus, become unduly large. A remedy of this situation will be introduced in the following corollary.

(4) In the theorem, in fact, only $\beta_{1}$ needs to be chosen large enough provided the magnitudes of the reference input and, hence, $|\xi|$ are bounded away from zero. This fact can be easily seen from equation (40). In other words, in a task of tracking, determination of $\beta_{1}$ will be crucial to tune the systems performance.

(5) Usually, $\varepsilon$ is chosen to be very small to guarantee the tracking accuracy. However, under practical digital implementation, $\varepsilon$ is chosen only reasonably small to prevent excessive switching in the input $u_{p}$ due to the finite-step-size numerical integration algorithm.

In the standard adaptive literature, it is well known that the parameter error vector $\phi$ in general does not converge to zero and will do so only when the regressor vector $w$ or, equivalently, $\xi$ is persistently exciting (Annaswamy and Narendra, 1988). From the previous results, $\beta_{1}$ has to be chosen to satisfy $\beta_{1} \geq \sup _{t \geq t_{f c}}|\phi(t)|$ in order to obtain the well-behaved transient response and nice tracking property regardless of the type of tasks, i.e. either stabilization or tracking. Then, it follows from the previous remark (3) that the resulting input may have unacceptably large magnitude to meet that requirement. However, alleviation of such a situation may be attained if $|\phi(t)|$ does converge to a fairly small sized interval asymptotically. The following corollary will state the condition under which this is true.

Corollary. (Economical control input due to PE condition.) Consider the previous problem in Theorem 1 with the same hypotheses. If, furthermore, $w$ is persistently exciting and $\min _{t}|\xi(t)| \geqslant m_{\xi}>0$, then the level of magnitude of $\beta_{1}$ to ensure the results of Theorem 1 is a class $K$ function of both $\varepsilon$ and $\Delta t$. 
By applying an argument similar to that in Ioannou and Sun (1990) and equation (40), previous remark (4), we can show that the above corollary is valid (Fu, 1992).

It is also a well-known fact in the adaptive literature that the convergence of the parameter error vector $\phi$ becomes slow when the order of the plant increases. The consequence of such slow convergence is, then, that $t_{f c}$ after which equation (41) is satisfied will be large and, hence, that the convergence of $e_{0}$ will be slowed down as well. A remedy of this is to forsake the parameter adaptation process and fix $\theta$ at the best estimates of $\theta^{*}$ that one can get and adopt the same control law. The following corollary will summarize the resulting property.

Corollary 2. (Fast output convergence without parameter adaptation.) Consider the same problem in Corollary 1 but fix $\theta$ at the best estimates of $\theta^{*}$ that one can get. Then all the results in Theorem 1 will remain valid provided $\beta_{1} \geq|\phi|=\left|\theta-\theta^{*}\right|$.

The proof is quite straightforward if we replace the Lyapunov function $V(e, \phi)$ by $V(e)$, i.e.

$$
V(e)=\frac{1}{2} e^{T} P_{e} e,
$$

and hence, is omitted here.

Remark. Usually, if the magnitude of the control input is severely constrained, then Corollary 1 will suffice to solve the control problem and incidentally meet the control limitation. However, the slow convergence of the output error may not be desirable due to the limited rate of convergence of the parameter errors $\phi$. Under this circumstance, a remedy is to use some prior information about $\theta^{*}$ such as the knowledge of $\theta_{\min }, \theta_{\max } \in R^{2 n}$ where $\theta_{\min } \leq$ $\theta^{*} \leqslant \theta_{\max }$, and, then, apply the mechanism of Corollary 2. As a result, both economical input design and fast output convergence are achieved simultaneously presuming the size of the interval $\left[\theta_{\min }, \theta_{\max }\right]$ is much smaller than $\theta_{0}$.

\section{ROBUSTNESS TO UNMODELLED DYNAMICS AND OUTPUT DISTURBANCES}

In this section, we will examine the robustness property of the adaptive controller in the non-ideal case where $\Delta \hat{P}_{1}(s), \Delta \hat{P}_{2}(s)$, and $\zeta_{0}$ are present. Recall that the error system in this case is given by equations (16)-(17), and the stability of the overall adaptive system will now require the uniform boundedness of $x_{\zeta}$ in addition to that of $e, w$, and $\theta$. Similar to the previous analysis, we first investigate whether all signals belong to the extended $L_{\infty}$ space. Therefore, we assume that there exists finite $T>0$ such that some signal grows unbounded at $t=T$ and, hence, for all $t \in[0, T)$ the system given by equations (16)-(17) can be rewritten in the following form:

$$
\begin{gathered}
\dot{e}=A_{m} e+b_{m}\left(\phi^{T \xi}+f\left(\hat{e}_{0}\right) \pi+\eta_{0}\right. \\
\left.+\mu \eta_{i}+\Delta\left(\hat{e}_{0}\right)\right), \\
\hat{e}_{0}=c_{m}^{T} e+\zeta_{0}=e_{0}+\zeta_{0}, \\
\dot{x}_{\zeta}=A_{\zeta} x_{\zeta}+b_{\zeta}\left(\theta^{T} \xi+f\left(\hat{e}_{0}\right) \pi(|\xi|)+\Delta\left(\hat{e}_{0}\right)\right), \\
\eta_{i}=c_{\zeta}^{T} x_{\zeta}+d_{\zeta}\left(\theta^{T} \xi+f\left(\hat{e}_{0}\right) \pi(|\xi|)+\Delta\left(\hat{e}_{0}\right)\right) .
\end{gathered}
$$

Construct a function similar to that in (26) as follows:

$V\left(e, \phi, x_{\zeta}\right)=\frac{1}{2} e^{T} P_{e} e+\frac{1}{2} \phi^{T} \Gamma^{-1} \phi+\frac{1}{2} \mu x_{\zeta}^{T} P_{\zeta} x_{\zeta}$,

where $P_{\zeta}(>0)$ satisfies

$$
-\left(A_{\zeta}^{T} P_{\zeta}+P_{\zeta} A_{\zeta}\right)=2 Q_{\zeta}(>0)
$$

and differentiate it along the solution trajectories of equations (18), (44)-(45) to obtain the following:

$$
\begin{aligned}
\dot{V}= & -\left(e^{T} Q_{e} e+\mu x_{\zeta}^{T} Q_{\zeta} x_{\zeta}+\sigma \phi^{T} \theta\right) \\
& +\left\{e_{0} f\left(\hat{e}_{0}\right) \pi+e_{0} \mu\left(c_{\zeta}^{T} x_{\zeta}+d_{\zeta}\left(\theta^{T} \xi+f\left(\hat{e}_{0}\right) \pi\right.\right.\right. \\
& \left.\left.\left.+\Delta\left(\hat{e}_{0}\right)\right)\right)+e_{0} \eta_{0}+e_{0} \Delta\left(\hat{e}_{0}\right)\right\} \\
& -\left(\zeta_{0} \phi^{T \xi}-\mu x_{\zeta}^{T} P_{\zeta} b_{\zeta}\left(\theta^{T} \xi+f\left(\hat{e}_{0}\right) \pi+\Delta\left(\hat{e}_{0}\right)\right)\right),
\end{aligned}
$$

which is to be examined under the circumstance where $\left|\hat{e}_{0}\right|>\varepsilon$. Let $p_{\zeta 1}, p_{\zeta 2}>0$ and $q_{\zeta 1}, q_{\zeta 2}>0$ be such that $p_{\zeta} I \leq P_{\zeta} \leq p_{\zeta 2} I$ and $q_{\zeta 1} I \leq Q_{\zeta} \leq$ $q_{\zeta 2} I$.

Substitution of equations (20) and (29) into (48) readily leads to the following:

$$
\begin{aligned}
\dot{V} \leq & -\left(q_{e_{1}}|e|^{2}+\mu q_{\xi_{1}}\left|x_{\zeta}\right|^{2}+\sigma \phi^{T} \theta\right) \\
& -\left|\hat{e}_{0}\right|\left\{\left(\left(1-\mu k_{1}\right) \beta_{1}\right)|\xi|\right. \\
& \left.+\left(\left(1-\mu k_{1}\right) \beta_{2}-\left|\eta_{0}\right|-\left(1+\mu k_{1}\right) \varepsilon^{\prime}(t)\right)\right\} \\
& +\mu\left(k_{3}|e|\left|x_{\zeta}\right|+k_{4}\left(\beta_{1}+|\theta|\right)\left|x_{\zeta}\right||\xi|\right. \\
& \left.+k_{2}|\theta||e||\xi|+k_{4}\left(\beta_{2}+\varepsilon^{\prime}(t)\right)\left|x_{\xi}\right|\right) \\
& +\left|\zeta_{0}\right|\left\{\left(\left(1+\mu k_{1}\right) \beta_{1}+\left(|\theta|+\theta_{0}\right)\right)|\xi|\right. \\
& \left.+\left(\left(1+\mu k_{1}\right) \beta_{2}+\left|\eta_{0}\right|+\left(1+\mu k_{1}\right) \varepsilon^{\prime}(t)\right)\right\},
\end{aligned}
$$

where $k_{1}=\left|d_{\zeta}\right|, k_{2}=\left|c_{m}\right|\left|d_{\zeta}\right|, k_{3}=\left|c_{m}\right|\left|c_{\zeta}\right|$, and $k_{4}=\left|P_{\zeta} b_{\zeta}\right|$. It can be verified (Fu, 1992) that there exist $\mu_{2}, \rho_{1}>0$ such that for all $\mu \in\left[0, \mu_{2}\right]$, for all $\rho \in\left[0, \rho_{1}\right]$, and for some $t_{1} \geq 0$

$$
\begin{aligned}
\dot{V} \leq & -\left(\bar{q}_{e 1}|e|^{2}+\mu \bar{q}_{{ }_{1} 1}\left|x_{\zeta}\right|^{2}\right)+k_{5} \mu\left|x_{\zeta}\right| \\
& +\left(k_{6} \mu^{2 / 3}|\xi|^{2}+k_{7} \rho|\xi|+k_{8} \rho\right),
\end{aligned}
$$

for some $\bar{q}_{e 1}, \bar{q}_{\zeta 1}, k_{i}>0, \quad i=5,6,7,8$, after $t \geq t_{1}$ when $\theta(t)$ turns out to be uniformly bounded in $t$, i.e. there exists $\theta_{\max }>0$ such that 
$|\theta(t)| \leq \theta_{\max }$ for all $t \in[0, T)$, but

$$
\begin{aligned}
\dot{V} \leq & -\left(\bar{q}_{e 1}|e|^{2}+\mu \bar{q}_{\zeta 1}\left|x_{\zeta 1}\right|^{2}+\sigma_{1}|\phi|^{2}\right)+k_{5} \mu\left|x_{\zeta}\right| \\
& +\left\{-\left(\sigma_{2}|\phi|^{2}-k_{9} \mu^{2 / 3}-\frac{\rho}{2}\right)|\xi|^{2}\right. \\
& \left.+k_{10} \rho|\xi|+k_{8} \rho\right\}
\end{aligned}
$$

for some $\sigma_{1}, \sigma_{2}, k_{9}, k_{10}>0$ when $|\theta(t)|$ grows gradually unbounded so that there exists $\theta_{L}$ $\left(\geq 3 \theta_{0}\right)$ and $t_{L}(\geq 0)$, to be determined later, such that $\left|\theta\left(t_{L}\right)\right| \geq \theta_{L}$. However, later we will show that the latter case will never occur.

Now, an upper bound and a lower bound on $V$ in equation (46) can be estimated as:

$$
\begin{aligned}
\frac{p_{e 1}}{2}|e|^{2}+\frac{\mu p_{\zeta 1}}{2} & \left|x_{\zeta}\right|^{2}+\frac{g_{1}}{2}|\phi|^{2} \leq V\left(e, \phi, x_{\zeta}\right) \\
& \leq \frac{p_{e 2}}{2}|e|^{2}+\frac{\mu p_{\zeta 2}}{2}\left|x_{\zeta}\right|^{2}+\frac{g_{2}}{2}|\phi|^{2} .
\end{aligned}
$$

Let $\mu_{3}=\min \left(\mu_{1}, \mu_{2}\right)$, where $\mu_{1}$ is given in Lemma A3 in the Appendix. Then, in the case where $|\theta(t)| \leq \theta_{\max }$ for all $t \in[0, T)$, we have $\left\|\hat{\xi}_{t}\right\|_{\infty} \leq \bar{\alpha}_{6}\left\|e_{t}\right\|_{\infty}+\alpha_{7}$, where $\bar{\alpha}_{6}=\alpha_{6}\left|c_{m}\right|$, for all $\mu \in\left[0, \mu_{3}\right]$ as a result of Lemma $A 4$. Thus, by adding $\sigma_{1}|\phi|^{2}$ to the first bracket and $\sigma_{1}\left(\theta_{\max }+\right.$ $\left.\theta_{0}\right)^{2}$ to the second bracket on the right hand side of equation (50), we readily arrive at the following expression:

$$
\begin{aligned}
\dot{V} \leq & -k_{11} V+k_{12} \mu^{1 / 2} V^{1 / 2} \\
& +\left(k_{6} \mu^{2 / 3}|\xi|^{2}+k_{7} \rho|\xi|+k_{13}\right) \\
\leq & -k_{11} V+\left(K_{1}(\mu)\left\|V_{t}\right\|_{\infty}\right. \\
& \left.+K_{2}(\mu, \rho)\left\|V_{t}\right\|_{\infty}^{1 / 2}+K_{3}(\mu, \rho)\right),
\end{aligned}
$$

for some $k_{i}>0, i=11,12,13$, and for some positive functions $K_{1}(\mu), K_{2}(\mu, \rho), K_{3}(\mu, \rho)$, where above all $K_{1}(\mu)$ is a class $K$ function of $\mu$.

On the other hand, in the case where $|\theta(t)| \geq \theta_{L} \geq 3 \theta_{0}$ when $t=t_{L}$, equation (51) can be expressed as:

$$
\begin{aligned}
\dot{V} \leq- & k_{11} V+k_{12} \mu^{1 / 2} V^{1 / 2} \\
& +\left\{-\left(\sigma_{2}|\phi|^{2}-\left(k_{9} \mu^{2 / 3}+\frac{\rho}{2}\right)\right)|\xi|^{2}\right. \\
& \left.+k_{10} \rho|\xi|+k_{8} \rho\right\}
\end{aligned}
$$

for all $t \in\left[t_{L}, T\right)$.

Now we are ready to state the robustness property of the previously proposed scheme against unmodelled dynamics and output disturbance.

Theorem 2. (Robustness property.) Given the system considered in Theorem 1 but in the non-ideal case, satisfying Assumptions (A1)-
(A6). Then, there exist $\mu^{*}>0$ and $\rho^{*}>0$ such that, for all $\mu \in\left[0, \mu^{*}\right]$ and $\rho \in\left[0, \rho^{*}\right]$, all signals inside the closed loop system remain uniformly bounded in $t$ provided that $\Delta t$ is sufficiently small. Furthermore, the output error $e_{0}$ will converge to a residual set whose size can be written as an explicit class $K$ function of $\mu, \rho$, and $\varepsilon$ provided the control parameters $\beta_{1}$ and $\beta_{2}$ are chosen properly large.

Proof of Theorem 2. Let us first assume that $e_{0}$ grows unbounded at some finite time $t=T$ and, hence, $\hat{e}_{0}$ is bounded away from the interval $[-\varepsilon, \varepsilon]$. Under this circumstance, the proof will proceed in two directions, namely, one is to assume $\theta(t)$ is uniformly bounded, i.e. $|\theta(t)| \leq$ $\theta_{\max }$ for all $t \in[0, T)$, and the other is to assume that $\theta(t)$ grow also unbounded so that $|\theta(t)| \geq \theta_{L} \geq 3 \theta_{0}$ at $t=t_{L}(<T)$.

In the first direction, the Lyapunov function $V$ has to satisfy the dynamic constraint given by equation (53). Thus, using Bellman-Gronwall lemma, $V$ can be shown to satisfy the following algebraic constraints:

$$
\begin{aligned}
V(t) \leq & \left\|V_{t}\right\|_{\infty} \\
\leq & \frac{1}{k_{11}}\left(K_{1}(\mu)\left\|V_{t}\right\|_{\infty}+K_{2}(\mu, \rho)\left\|V_{t}\right\|_{\infty}^{1 / 2}\right. \\
& \left.+K_{3}(\mu, \rho)\right)+\varepsilon^{\prime}(t) .
\end{aligned}
$$

Since $K_{1}(\mu)$ is a class $K$ function of $\mu$, there exists $\mu^{*}$, satisfying $0 \leq \mu^{*} \leq \mu_{3}$, such that $k_{11}^{-1} K_{1}\left(\mu^{*}\right)<1$ and, hence,

$$
\begin{aligned}
& V(t) \leq\left\|V_{t}\right\|_{\infty} \\
& \leq \frac{\left(k_{11}^{-1} K_{2}+\sqrt{\left(k_{11}^{-1} K_{2}^{2}+4\left(1-k_{11}^{-1} K_{1}\right)\left(k_{11}^{-1} K_{3}+\varepsilon^{\prime}\right)\right.}\right.}{4\left(1-k_{11}^{-1} K_{1}\right)^{2}},
\end{aligned}
$$

so long as $\mu \in\left[0, \mu^{*}\right]$. Whereas in the second direction where $\theta(t)$ grows unbounded, the dynamic constraint on $V$ becomes equation (54). Now let $\rho^{*}=\rho_{1}$ as defined before. Then, it can be verified that, if $\theta_{L}$ is defined to be

$$
\theta_{L}=\max \left(\sqrt{\frac{k_{9} \mu^{* 2 / 3}+\rho^{*} / 2}{\sigma_{2}}}+2 \theta_{0}, 3 \theta_{0}\right)
$$

then, for all $\mu \in\left[0, \mu^{*}\right]$ and $\rho \in\left[0, \rho^{*}\right]$, equation (54) can be put into an equivalent form:

$$
\dot{V} \leq-k_{11} V+k_{12} \mu^{1 / 2} V^{1 / 2}+k_{14} \text {, }
$$

for some $k_{14}>0$, which readily leads to the following result:

$$
\begin{aligned}
& V(t) \leq\left\|V_{t}\right\|_{\infty} \\
& \leq \frac{1}{4}\left(\left(k_{11}^{-1} k_{12} \mu^{1 / 2}+\sqrt{\left(k_{11}^{-1} k_{12}\right)^{2} \mu+4 k_{11}^{-1} k_{15}}\right)^{2} .\right.
\end{aligned}
$$

From equations (56) and (59), it is clear that $e_{0}$, 
in fact, can not escape in finite time $t=T$ and, hence, it belongs to the extended $L_{\infty}$ space. This again immediately implies that $\phi(\cdot) \in L_{\infty e}^{2 n}$ and $\xi(\cdot) \in L_{\infty e}^{2 n}$ from Lemma $A 1$ and Lemma A2, respectively. In consequence, the expression of the overall system given by equations (44)-(45) is, then, valid for all $t \geq 0$.

Now, if one further evaluates the derivative of $V$ when $\left|\hat{e}_{0}\right|<\varepsilon$, then it can be verified (Fu, 1992) that, though $k_{12}, k_{14}, K_{2}$, and $K_{3}$ will have dependence on $t$ and $\Delta t$, they are uniformly bounded provided all the signals $e, \phi, w$, and $x_{\zeta}$ are uniformly bounded for all $t \geq 0$. Therefore, using the argument in the proof of Theorem 1, it can be easily seen that for sufficiently small $\Delta t$ all the aforementioned properties remain valid.

To show the size of the residual set of $e_{0}$ is a class $K$ function of $\mu, \rho$, and $\varepsilon$, we differentiate the signal $\frac{e_{0}^{2}}{2}$ in the following as:

$$
\begin{aligned}
e_{0} \dot{e}_{0}= & e_{0}\left(c_{m}^{T} A_{m} e+k_{p}\left(\phi^{T} \xi+f\left(\hat{e}_{0}\right) \pi\right.\right. \\
& \left.\left.+\Delta\left(\hat{e}_{0}\right)+\eta_{0}+\mu \eta_{i}\right)\right),
\end{aligned}
$$

so that when $\left|\hat{e}_{0}\right|>\varepsilon$, we have

$$
\begin{aligned}
\frac{1}{2} \frac{\mathrm{d}}{\mathrm{d} t}\left|e_{0}\right|^{2} \leq- & \left|e_{0}\right|\left(\left(k_{p} \beta_{2}-\left\|c_{m}^{T} A_{m}\right\||e|-k_{p} \varepsilon^{\prime}(t)\right)\right. \\
& \left.+k_{p}\left(\beta_{1}-|\phi|\right)|\xi|\right) \\
& +k_{p}\left|e_{0}\right|\left(b_{p} \rho+\left|\eta_{i}\right| \mu\right)+2 k_{p} \rho \pi(|\xi|)
\end{aligned}
$$

where we use the fact that $e_{0} \operatorname{sgn}\left(\hat{e}_{0}\right) \geq\left|e_{0}\right|-2 \rho$ in the above derivation. Suppose now the control parameters $\beta_{1}$ and $\beta_{2}$ are chosen such that for all $t \geq t_{f c}$, for some finite $t_{f c} \geq 0$,

$$
\begin{aligned}
& \beta_{1} \geq \max _{t \geq t_{f c}}|\phi(t)|, \\
& \beta_{2} \geq \max _{t \geq t_{f c}} \frac{1}{k_{p}}\left\|c_{m}^{T} A_{m}\right\||e(t)|+\bar{\delta} \Delta t,
\end{aligned}
$$

as required in Theorem 1 , then after some re-assignment of notation, equation (61) can be expressed as:

$$
\frac{1}{2} \frac{\mathrm{d}}{\mathrm{d} t}\left|e_{0}\right|^{2} \leq-\bar{\beta}_{3}\left|e_{0}\right|+K_{4}(\mu, \rho)
$$

where

$$
\begin{aligned}
K_{4}(\mu, \rho)= & k_{p}\left|c_{m}\right|\left\|e_{t}\right\|_{\infty}\left(b_{\rho} \rho+\left\|\eta_{i t}\right\|_{\infty}\right) \\
& +2 k_{p} \rho\left\|\tau_{t}\right\|_{\infty} .
\end{aligned}
$$

Obviously, $\quad K_{4}(0, \rho)=O(\rho) \quad$ and $\quad K_{4}(\mu, 0)=$ $O(\mu)$. Finally, since $\left|\hat{e}_{0}\right|>\left|e_{0}\right|-\rho$, whenever $\left|e_{0}\right|>\rho+\varepsilon$, we have $\left|\hat{e}_{0}\right| \geq \varepsilon$ and, hence, equation (63) can be concluded. This implies that $e_{0}$ will eventually converge into a residual set whose size is a class $K$ function of $\mu, \rho$, and $\varepsilon$. This completes the proof.

Q.E.D.
6. NUMERICAL SIMULATION EXAMPLES

In this section, we will illustrate the effectiveness of the proposed new MRAC scheme by performing the following computer simulation example.

Consider a plant described by

$$
\hat{P}_{u}(s)=\frac{2}{(s+1)(s-3)}\left(1+\mu \Delta \hat{P}_{1}(s)\right)+\mu \Delta \hat{P}_{2}(s),
$$

where

$$
\begin{aligned}
& \Delta \hat{P}_{1}(s)=\frac{1}{s+12}, \\
& \Delta \hat{P}_{2}(s)=\frac{2}{s+10},
\end{aligned}
$$

and the reference model is given by

$$
\hat{M}(s)=\frac{1}{(s+2)(s+3)} .
$$

When $\mu=0$, it can be verified that the true parameter vector $\theta^{*}=[0.5,-1.4,22.4,-29]^{T}$ and $\left|\theta^{*}\right|=36.67$. Therefore, a reasonable choice of $\theta_{0}$ in equation (18) is $\theta_{0}=40$. Let $\hat{L}(s)=(s+2.5)$ so as to make $\hat{M}(s) \hat{L}(s)$ SPR and let reference input $r=3 \sin \left(2^{*} t\right)$ in the task of tracking. The numerical values for the parameters in equations (18)-(20) are given by: $\sigma_{0}=3, \Gamma=5, \Delta t=0.001$, and $\varepsilon=0.2$. Let $\mu=0.01$ and $\zeta_{0}=0.1^{*} \cos (t)$. First of all, the conventional MRAC schemes (Narendra and Valavani, 1978; Narendra et al., 1980; Ioannou and Tsakalis, 1986) $\left(\beta_{1}=\beta_{2}=0\right)$ are applied to this example and the resulting signal trajectories are shown in Fig. 2. Next, we apply the
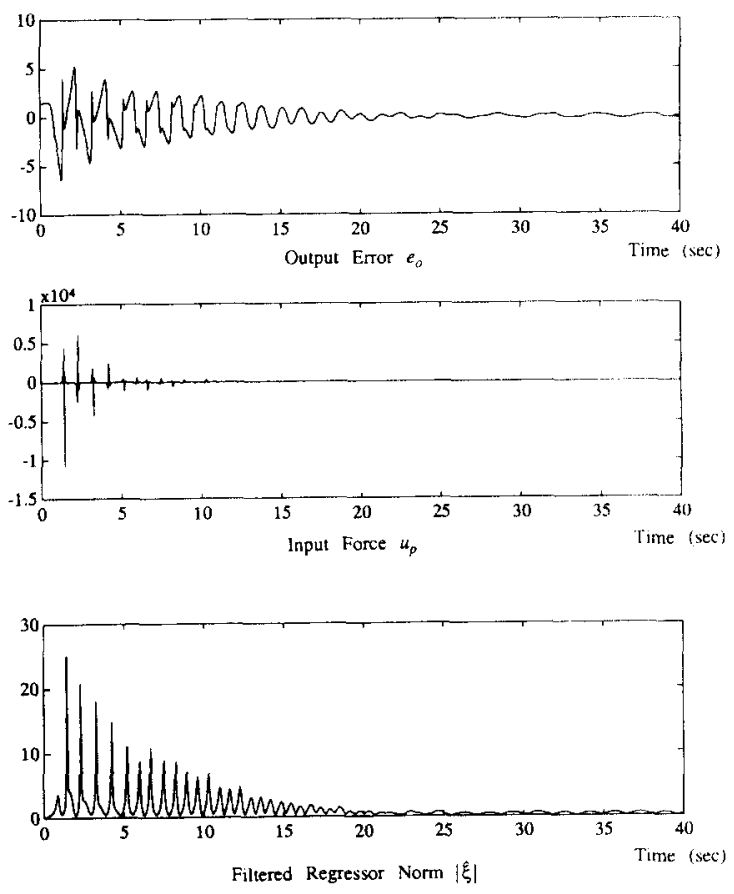

FIG. 2. Conventional scheme. 

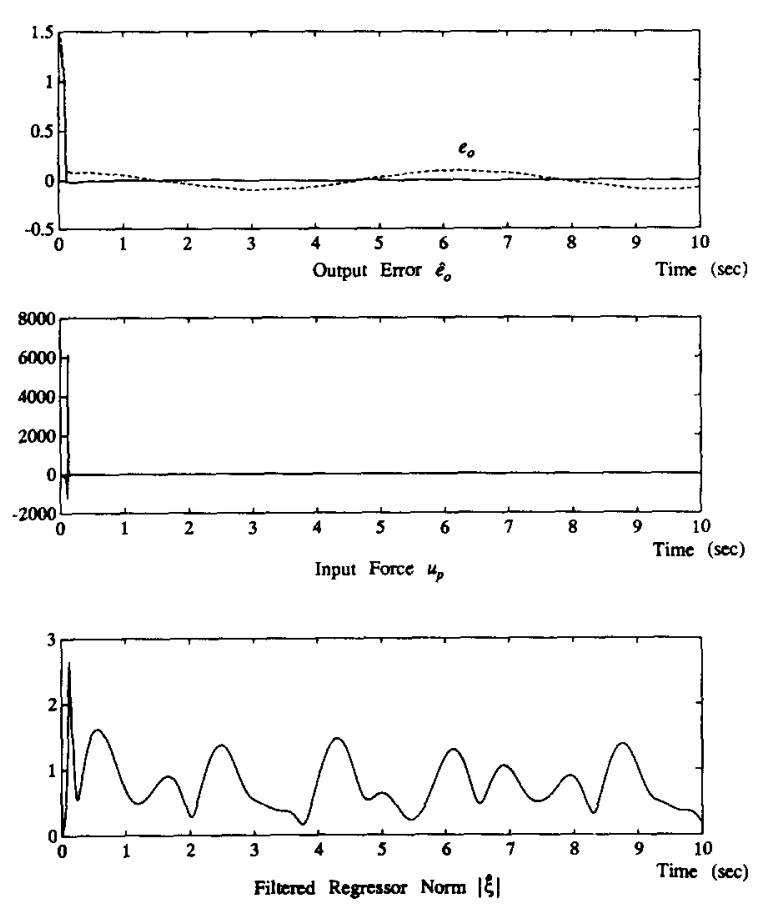

FIG. 3. $r=3 \sin \left(2^{*} t\right), \beta_{1}=40, \beta_{2}=1$.

proposed scheme in different situations: (i) Suppose the knowledge about $\theta^{*}$ is only the upper bound of its norm, i.e. $\theta_{0}$. Then, we choose $\beta_{1}=40$ and let $\beta_{2}=1$, following Remarks (3) and (4) after the proof of Theorem 1 in Section 4. Figure 3 illustrates the resulting signal trajectories. (ii) Suppose the reference input is enriched so that $r=3 \sin \left(2^{*} t\right)+$ $2 \sin \left(4^{*} t\right)$. Then, $\beta_{1}$ can be greatly reduced so that $\beta_{1}=2$ but $\beta_{2}$ remains the same, based on the result of Corollary 1 . The resulting signal
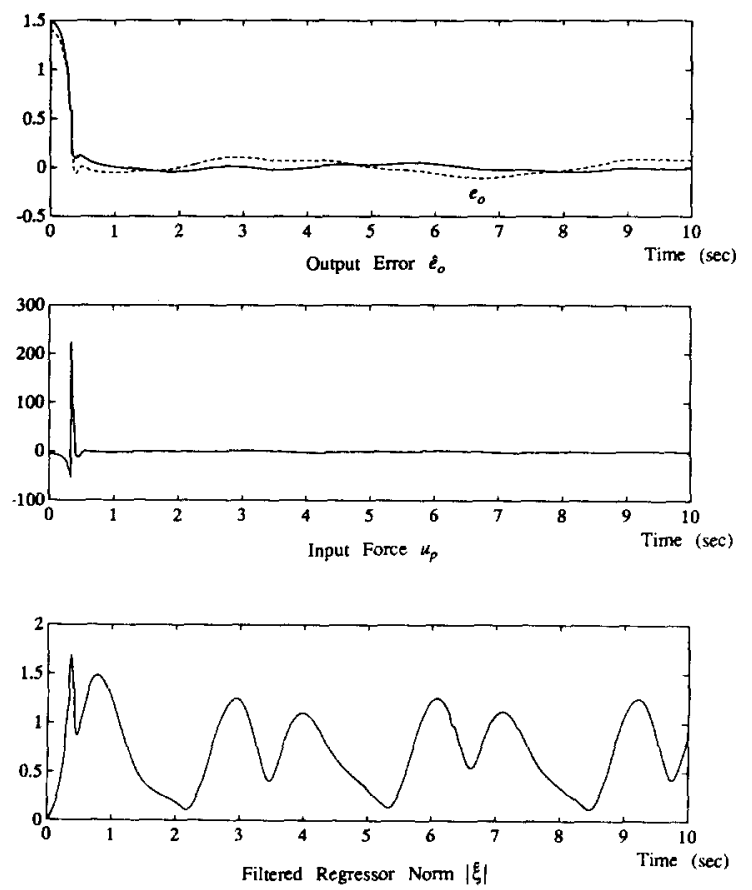

FIG. 4. $r=3 \sin \left(2^{*} t\right)+2 \sin \left(4^{*} t\right), \beta_{1}=2, \beta_{2}=1$.
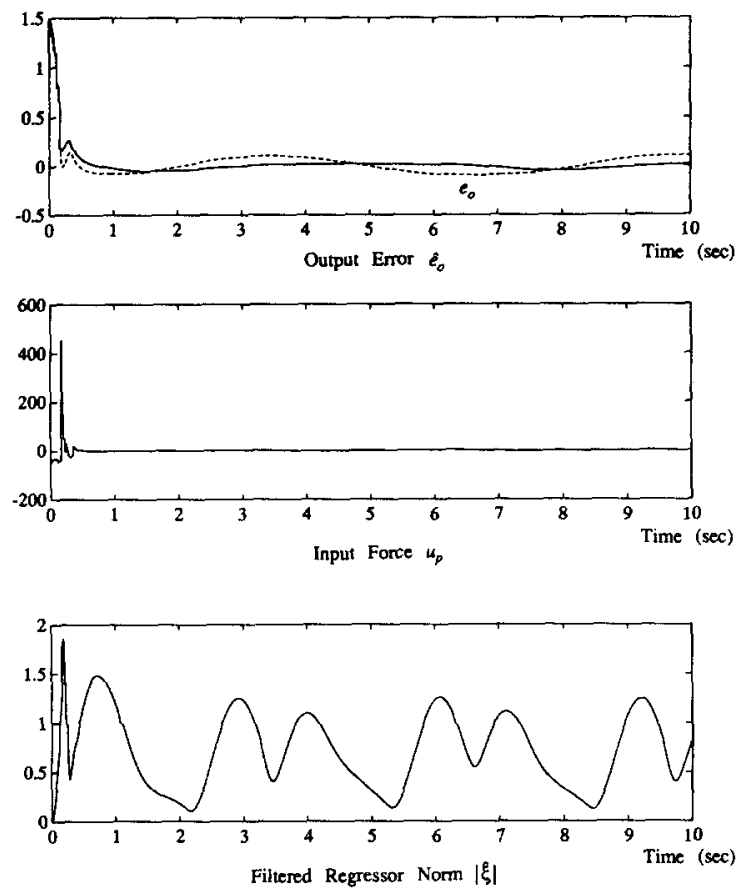

FIG. 5. $\theta_{\min }=[0.1,-2,22,-30]^{T}$ and $\theta_{\max }=[0.9,-1,23,-$ $28]^{T}, \theta=\theta_{\min }$ and $r=3 \sin \left(2^{*} t\right)$.

trajectories are provided in Fig. 4. (ii) Suppose the interval in which $\theta^{*}$ lies is $\left[\theta_{\min }, \theta_{\max }\right.$ ], where $\theta_{\min }=[0.1,-2,22,-30]^{T}$ and $\theta_{\max }=$ $[0.9,-1,23,-28]^{T}$. Then, from the remark after Corollary 2, we let $\theta=\theta_{\min }$ and choose $\beta_{1}=3$, since $\left|\theta_{\max }-\theta_{\min }\right|=2.58$, but let $\beta_{2}$ still remain the same. In Fig. 5 , the resulting signal trajectories are provided. (iv) Finally, just to show the drastic improvement of both the transient behavior and the tracking performance of the closed loop system by adding small
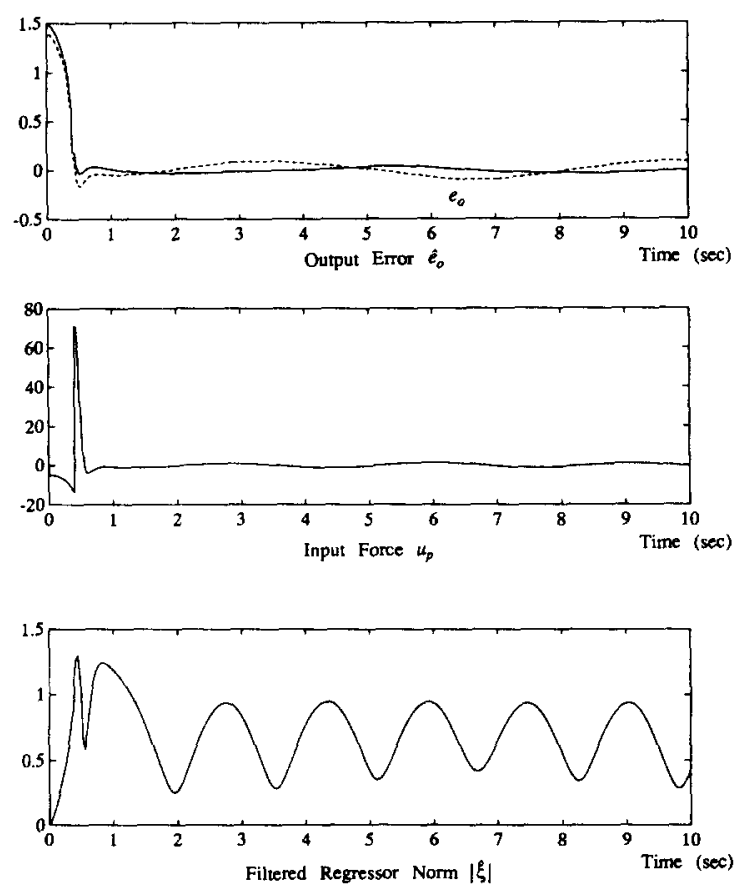

FIG. 6. $r=3 \sin \left(2^{*} t\right), \beta_{1}=0, \beta_{2}=2$. 

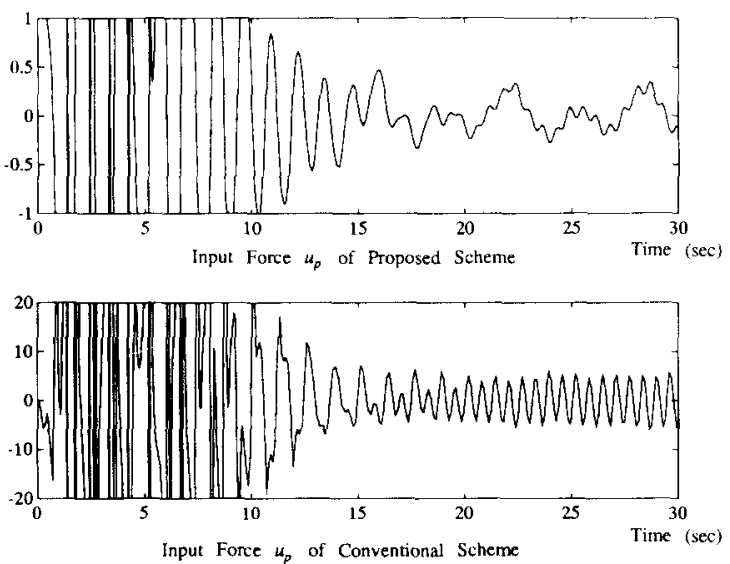

FIG. 7. Comparison between the conventional scheme and the proposed scheme.

quantity of $v_{p}$ into $u_{p}$ as given in (3.3), we let $\beta_{1}=0$ and $\beta_{2}=2$. The simulated signal trajectories are illustrated in Fig. 6, which seem to be much better behaved than those in Fig. 2. For interesting comparison, a close-up of the time evolutions of the input force $u_{p}$ applied in the conventional scheme and that in the current scheme in situation (iv) in Fig. 7 suggests that the steady state $u_{p}$ of the proposed scheme has smaller magnitude and evolves smoother with time than that of $u_{p}$ in the conventional schemes.

\section{CONCLUSIONS}

The paper presented a new robust MRAC scheme for SISO plants with relative degree two, which incorporated the similar VSD concept in Fu (1991) and was based on the modified MRAC scheme proposed in Narendra and Valavani (1978) particularly for the case with relative degree two. No assumption of PE condition is needed here in order to ensure the global stability of the overall system, in a way similar to $\sigma$ - (Ioannou and Kokotovic, 1984b; Ioannou, 1986; Ioannou and Tsakalis, 1986) and $e_{1^{-}}$ modification (Narendra and Annaswamy, 1987). Since the controller is a continuous one in nature, no Filippov's notion is used and hence, the input force is generally kept with a moderate level of magnitude and usually evolves with smoother time trajectory. The striking features of the proposed controller are the drastic improvement of the transient behavior and the tracking performance from the conventional schemes (Narendra and Valavani, 1978; Narendra et al., 1980; Ioannou and Tsakalis, 1986) which are clearly demonstrated in the simulation example.

In the proposed scheme, both parameter update process and the input interaction contributed to the stabilization of the overall system. Due to this sharing of load between two mechanisms in handling the parameter uncertainties, the magnitude of the resulting input force has been greatly reduced as opposed to those observed in Narendra and Valavani (1978), Narendra et al. (1980) or Fu (1991). Furthermore, if the reference input satisfies the PE condition or if the prior knowledge about the neighborhood of the system parameters is available, then the input magnitude can be reduced further or even the rate of convergence can be increased as well. In the presence of mild unmodelled dynamics and bounded output disturbance the proposed scheme was shown to be robust and the resulting tracking errors will fall into a residual set whose size has been related directly to the size of unmodelled dynamics and of output disturbances.

\section{REFERENCES}

Ambrosino, G., G. Celentano and F. Garofalo (1984). Variable structure model reference adaptive control systems. Int. J. Control, 39, 1339-1349.

Annaswamy, A. M. and K. S. Narendra (1988). Stable Adaptive Systems. Prentice-Hall, Englewood Cliffs, NJ.

Anderson, B. D. O., R. R. Bitmead, C. R. Johnson, P. V. Kokotovic, R. L. Kosut, I. M. Y. Mareels, L. Praly and B. D. Riedle (1986). Stability of Adaptive Systems, Passivity, and Averaging and Analysis. MIT Press, Cambridge, MA.

Ástrőm, K. J. (1935). Interaction between excitation and unmodelled dynamics. IEEE Trans. on Aut. Contro!, AC-30, 889-891.

Bodson, M. (1986). Stability, convergence, and robustness of adaptive systems, Ph.D. Dissertation, University of California, Berkeley.

Bodson, M. and S. S. Sastry (1984). Small signal I/O stability of nonlinear control systems: application to the robustness of a MRAC scheme, Memorandum, No UCB/ERL M84/70, Electronics Research Laboratory, University of California, Berkeley.

Bodson, M. and S. S. Sastry (1989). Adaptive Control: Stability, Convergence, and Robustness. Prentice-Hall, Englewood Cliffs, NJ

Chen, Z. J. and P. A. Cook (1984). Robustness of mode reference adaptive control systems with unmodelled dynamics. Int. J. Control, 39, 201-214.

Desoer, C. A. and M. Vidyasagar (1975). Feedback Systems: Input-output Properties. Academic Press, New York.

Egardt, B. (1979). Stability of Adaptive Controllers. Springer-Verlag, New York.

Egardt, B. (1980). Stability analysis of adaptive control systems with disturbances. Proc. JACC, San Francisco, CA.

Fu, L. C. (1991). A new approach to robust model reference adaptive control for a class of plants. Int. J. Control, 53, 1359-1375.

Fu, L. C. (1992). A new robust model reference adaptive control using variable structure design for plants with relative degree two. Technical Report, NTUEE 92-1, Department of Electrical Engineering, National Taiwan University, Taipei, Taiwan, R.O.C.

Fu, L. C. and S. S. Sastry (1987a). Frequency domain analysis and synthesis techniques for adaptive systems, Memorandum, No. UCB/ERL M87/58, Electronics Research Laboratory, University of California, Berkeley.

Fu, L. C. and S. S. Sastry (1987b). Slow drift instability in model reference adaptive systems-an averaging analysis. Int. J. Control, 45, 503-527.

Hsu, Liu (1990). Variable structure model-reference adaptive control (VS-MRAC) using only input and output 
measurements: the general case. IEEE Trans. Aut. Control, 35, 1238-1243.

Ioannou, P. A. (1986). Robust adaptive controller with zero residual tracking errors. IEEE Trans. Aut. Control, AC-31, 773-776.

Ioannou, P. A. and P. V. Kokotovic (1984a). Instability analysis and improvement of robustness of adaptive control. Automatica, 20, 583-594.

Ioannou, P. A. and P. V. Kokotovic, (1984b). Robust redesign of adaptive control. IEEE Trans. on Aut. Control. AC-29, 202-211.

Ioannou, P. A. and K. S. Tsakalis (1986). A robust direct adaptive control. IEEE Trans. Aut. Control, AC-31, 1033-1043.

Ioannou, P. A. and J. Sun (1990). Parameter Convergence of modified adaptive laws with persistent excitation. Proc. American Control Conf., pp. 565-566.

Kokotovic, P. V., B. Riedle and L. Praly (1985). On a stability criterion for continuous slow adaptation. System and Control Letters, 6, 7-14.

Kosut, R. L. and C. R. Johnson (1984). An input-output view of robustness in adaptive control. Automatica, 20, $569-581$

Kosut, R. L. and B. Friedlander (1985). Robust adaptive control: conditions for global stability. IEEE Trans. Aut. Control, AC-30, 610-624.

Krause, J., M. Athans, S. S. Sastry and L. Valavani (1983). Robustness studies in adaptive control. Proc. 22nd IEEE Conf. on Decision and Control.

Kreisselmeier, G. and B. D. O. Anderson (1986). Robust model reference adaptive control. IEEE Trans. Aut. Control, AC-31, 127-133.

Kreisselmeier, G. and K. S. Narendra (1982). Stable model reference adaptive control in the presence of bounded disturbances. IEEE Trans. Aut. Control, AC-27, 11691175.

Narendra, K. S. and L. Valavani (1978). Stable adaptive controller design-direct control. IEEE Trans. Aut. Control, AC-23, 570-583.

Narendra, K. S. and A. M. Annaswamy (1987). A new adaptive law for robust adaptation without persistent excitation. IEEE Trans. Aut. Control, AC-32, 134-145.

Narendra, K. S., Y. H. Lin and L. Valavani (1980). Stable adaptive controller design, Part II: proof of stability. IEEE Trans. Aut. Control, AC-25, 440-448.

Ortega, R., L. Praly and Y. Tang (1987). Direct adaptive tuning or robust controllers with guaranteed stability properties. System and Control Letters, 321-326.

Peterson, B. B. and K. S. Narendra (1982). Bounded error adaptive control. IEEE Trans. Aut. Control, AC-27, 1161-1168.

Riedle, B. D. and P. V. Kokotovic (1985). A stabilityinstability boundary for disturbance-free slow adaptation and unmodelled dynamics. IEEE Trans. Aut. Control AC-30, 1027-1030.

Rohrs, C. E., L. Valavani, M. Athans and G. Stein (1985). Robustness of continuous-time adaptive control algorithm in the presence of unmodelled dynamics. IEEE Trans. Aut. Control, AC-30, 881-889.

Sastry, S. S. (1984). Model reference adaptive control: stability, parameter convergence and robustness. I.M.A.J. Control, and Inform., 1, 27-66.

Vidyasagar, M. (1978). Nonlinear Systems Analysis. Prentice-Hall, Englewood Cliffs, NJ.

\section{APPENDIX}

\section{Lemmas and proofs}

Lemma A1. Consider the parameter update law given by equation (18). Then, there exist $\alpha_{1}, \alpha_{2}>0$ such that

$$
\left\|\phi_{t}\right\|_{\infty} \leq \alpha_{1}\left\|\hat{e}_{o t}\right\|_{\infty}+\alpha_{2} \text {. }
$$

Proof of Lemma A1. With loss of generality, we only need to consider the case where $|\theta|>2 \theta_{0}$. Therefore, equation (18) becomes

$$
\dot{\theta}=-\Gamma\left(\hat{e}_{0} \hat{\xi}+\sigma_{0}\left(1+|\hat{\xi}|^{2}\right) \theta\right)
$$

Define a Lyapunov function $V_{\theta}=\frac{1}{2} \theta^{T} \Gamma^{-1} \theta$ and, then, take its time derivative along the solution trajectories of (A.2) to obtain the following:

$$
\begin{aligned}
\frac{\mathrm{d}}{\mathrm{d} t} V_{\theta} & =-\sigma_{0}|\theta|^{2}-\sigma_{0}|\theta|^{2}|\xi|^{2}-\hat{e}_{0} \theta^{T \xi} \\
& \leq-\frac{2 \sigma_{0}}{g_{2}} V_{\theta}+\frac{\left|\hat{e}_{0}\right|^{2}}{4 \sigma_{0}}-\sigma_{0}\left(|\theta||\xi|-\frac{\left|\hat{e}_{0}\right|}{2 \sigma_{0}}\right)^{2} \\
& \leq-\frac{2 \sigma_{0}}{g_{2}} V_{\theta}+\frac{\left|\hat{e}_{0}\right|^{2}}{4 \sigma_{0}},
\end{aligned}
$$

wit ere we use the fact that $g_{1} I \leq \Gamma^{-1} \leq g_{2} I$ for some $g_{1}, g_{2}>0$. Thus, by Bellman-Gronwall lemma, the bound on $\theta$ can be derived as:

$$
|\theta|^{2} \leq \frac{g_{2}}{4 \sigma_{0}^{2} g_{1}}\left\|\hat{e}_{0 t}\right\|_{\infty}^{2}+\varepsilon^{\prime}(t),
$$

where $\varepsilon^{\prime}(t)$ is due to the stable initial conditions. Let $\alpha_{1}=$ $\frac{1}{2 \sigma_{0}}\left(\frac{g_{2}}{g_{1}}\right)^{1 / 2}$ and $\alpha_{2}=\max _{t} \varepsilon^{\prime}(t)+\theta_{0}$. Then, it follows that

$$
\left\|\phi_{t}\right\|_{\infty} \leq\left\|\theta_{\ell}\right\|_{\infty}+\theta_{0} \leq \alpha_{1}\left\|\hat{e}_{0 t}\right\|_{\infty}+\alpha_{2} .
$$

This completes the proof.

Q.E.D.

Lemma A2. Consider the problem described in Section 2 and Section 3, satisfying Assumptions (A1)-(A6). Let the adaptive controller be designed according to Section 4 . If $e_{0}(\cdot) \in L_{\infty e}$, then $\hat{w}(\cdot) \in L_{\infty e}^{2 n}$ and, hence $\hat{\xi}(\cdot) \in L_{\infty e_{e}}^{2 n}$

Proof of Lemma A2. First of all, from the hypotheses and Lemma A1, we know that $\phi(\cdot) \in L_{\infty}^{2 n}$. To show that $\hat{w}(\cdot)$ and $\xi(\cdot)$ belong to the extended $L_{\infty}$ space, we assume the contrary, i.e. there exists $T>0$ such that $|\hat{w}(t)|$ grow unbounded at $t=T$, and prove that such an assumption will lead to a contradiction.

Let the compensator blocks $F_{1}$ and $F_{2}$ be realized by a controllable pair $(\Lambda, b)$, where $\Lambda \in R^{(n-1) \times(n-1)}$ is Hurwitz and $b \in R^{(n-1)}$, so that $\hat{F}_{1}(s)=\hat{F}_{2}(s)=(s I-\Lambda)^{-1} b$. Thus we have

$$
\begin{aligned}
& \dot{v}_{p}^{(1)}=\Lambda v_{p}^{(1)}+b u_{p}, \\
& \dot{v}_{p}^{(2)}=\Lambda v_{p}^{(2)}+b\left(y_{p}+\zeta_{0}\right),
\end{aligned}
$$

and, hence, $\xi_{1}$ and $\xi_{2}$ will satisfy

$$
\begin{aligned}
\dot{\xi}_{1} & =\Lambda \xi_{1}+b \hat{L}^{-1}\left(u_{p}\right)+\varepsilon^{\prime}(t) \\
& =\Lambda \xi_{1}+b\left(\theta^{T} \xi+\hat{L}^{-1}(s)\left(v_{p}\right)\right)+\varepsilon^{\prime}(t), \\
\dot{\xi}_{2} & =\Lambda \xi_{2}+b \hat{L}^{-1}\left(y_{p}+\xi_{0}\right)+\varepsilon^{\prime}(t) .
\end{aligned}
$$

It then follows from (A.7) that

$$
\left\|\xi_{2 t}\right\|_{\infty} \leq \gamma_{2}\left\|y_{p}\right\|_{\infty}+\gamma_{3}, \quad \text { for all } t \in[0, T]
$$

for some positive constants $\gamma_{2}$ and $\gamma_{3}$. Furthermore, as a result from equations (19), (30)-(31), a bound on $\hat{L}^{-1}\left(v_{p}\right)$ can be estimated as:

$$
\begin{aligned}
\left|\hat{L}^{-1}\left(v_{p}\right)\right| \leq & 2\left(1+\frac{1}{a \Delta t}\right) \beta_{1}\left\|\hat{\xi}_{t}\right\|_{\infty} \\
& +\beta_{2}^{\prime}\left(1+\frac{2}{a \Delta t}\right) \\
& +\gamma_{1}+\beta_{1} \hat{L}^{-1}(s)(|\hat{w}|) \\
= & \gamma_{4}\left\|\hat{\xi}_{t}\right\|_{\infty}+\gamma_{5}+\beta_{1} \hat{L}^{-1}(s)(|\hat{w}|),
\end{aligned}
$$

for some $\gamma_{4}, \gamma_{5}>0$. Denote $\hat{w}=\left(w_{(1)}, \ldots, w_{(2 n)}\right)^{T} \in R^{2 n}$ and $\hat{\xi}=\hat{L}^{-1}(\hat{w})=\left(\xi_{(1)}, \ldots, \xi_{(2 n)}\right)^{T} \in R^{2 n}$. Suppose for some $j, 1 \leq j \leq 2 n, w_{j}(t)$ grows unbounded at $t=T$. Then, there exists $t_{f} \geq 0$ such that $w_{(j)}(t)=\operatorname{sgn}\left(w_{(j)}\left(t_{f}\right)\right)\left|w_{(j)}(t)\right|$ for 
all $t \geq t_{f}$ and, hence,

$$
\begin{aligned}
\left|\xi_{(j)}(t)\right|= & \left|\int_{0}^{t} e^{-a(t-\tau)} w_{(j)}(\tau) \mathrm{d} \tau\right| \\
\geq & \int_{t_{f}}^{t} e^{-a(t-\tau)}\left|w_{(j)}(\tau)\right| \mathrm{d} \tau \\
& -\left|\int_{0}^{t_{f}} e^{-a(t-\tau)} w_{(j)} \mathrm{d} \tau\right| \\
= & \int_{0}^{t} e^{-a(t-\tau)}\left|w_{(j)}(\tau)\right| \mathrm{d} \tau \\
& -\left|\int_{0}^{t_{f}} e^{-a(t-\tau)} w_{(j)}(\tau) \mathrm{d} \tau\right| \\
& -\int_{0}^{t_{f}} e^{-a(t-\tau)}\left|w_{(j)}(\tau)\right| \mathrm{d} \tau \\
\geq & \hat{L}^{-1}(s)\left(\left|w_{(j)}\right|\right)(t)-\gamma_{6}
\end{aligned}
$$$$
\text { for all } t \in\left[t_{f}, T\right] \text {, }
$$

for some $\gamma_{6}>0$. On the other hand, if $w_{(j)}(t)$ is bounded on $[0, T]$, then (A.10) will be even more trivially satisfied. Therefore, with the help of the facts that

$$
|\hat{w}| \leq \sum_{j}\left|w_{(j)}\right| \text { and } \sum_{j}\left|\xi_{(j)}\right| \leq(2 n)^{1 / 2}|\hat{\xi}|
$$

we can readily deduce from (A.10) the following:

$$
\hat{L}^{-1}(s)(|\hat{w}|) \leq(2 n)^{1 / 2}|\hat{\xi}|+\gamma_{7},
$$

for some positive constant $\gamma_{7}$. Combining (A.9) with (A.12), we thus have

$$
\left|\hat{L}^{-1}\left(v_{p}\right)\right| \leq \bar{\gamma}_{4}\left\|\hat{\xi}_{t}\right\|_{\infty}+\bar{\gamma}_{5},
$$

where $\bar{\gamma}_{4}=\gamma_{4}+\beta_{1}(2 n)^{1 / 2}$ and $\bar{\gamma}_{5}=\gamma_{5}+\gamma_{7}$. Finally, by substitution of (A.13) into the estimate of the rate of change of $\xi_{1}$ in (A.7), we will have

$$
\begin{aligned}
\left|\dot{\xi}_{1}\right| \leq & \left(\left\|\Lambda+b C^{T}(t)\right\|+\bar{\gamma}_{4}\right)\left\|\xi_{1 t}\right\|_{\infty} \\
& +\left(\left\|b D^{T}(t)\right\|+\bar{\gamma}_{4}\right)\left\|\xi_{2 t}\right\|_{\infty} \\
& +\left(\left|b d_{0}(t)\right|+\bar{\gamma}_{4}\right)\left\|\left(\hat{L}^{-1}\left(y_{p}+\xi_{0}\right)\right)_{1}\right\|_{\infty} \\
& +\left\{\left(\left|b c_{0}(t)\right|+\bar{\gamma}_{4}\right)\left\|\left(\hat{L}^{-1}(r)\right)_{t}\right\|_{\infty}+|b| \bar{\gamma}_{5}+\left|\varepsilon^{\prime}(t)\right|\right\} \\
\leq & \alpha_{3}\left\|\xi_{1 t}\right\|_{\infty}+\alpha_{4}\left\|y_{p t}\right\|_{\infty}+\alpha_{5} \\
& \text { for all } t \in[0, T),
\end{aligned}
$$

where

$$
\begin{aligned}
\alpha_{3}= & \max _{t \geqslant 0}\left(\left\|\Lambda+b C^{T}(t)\right\|+\bar{\gamma}^{4}\right), \\
\alpha_{4}= & \max _{t \geq 0}\left(\left(\left\|b D^{T}(t)\right\|+\bar{\gamma}_{4}\right) \gamma_{2}+\left(\left|b d_{0}(t)\right|+\bar{\gamma}_{4}\right) \frac{1}{a}\right), \\
\alpha_{5}= & \max _{t \geq 0}\left(\left(\left|b c_{0}(t)\right|+\bar{\gamma}_{4}\right)\left|\hat{L}^{-1}(r)\right|+\left(|| b D^{T}(t) \|+\bar{\gamma}_{4}\right) \gamma_{3}\right. \\
& \left.+\left(\left|b d_{0}(t)\right|+\bar{\gamma}_{4}\right)\left(\left|\hat{L}^{-1}\left(\xi_{0}\right)\right|+\gamma_{1}\right)+\left|\varepsilon^{\prime}(t)\right|+|b| \bar{\gamma}_{5}\right) .
\end{aligned}
$$

Note that in the above derivations we have used the facts that

$$
\begin{aligned}
\left\|\hat{\xi}_{\ell}\right\|_{\infty} \leq & \left\|\left(\hat{L}^{-1}(r)\right)_{l}\right\|_{\infty}+\left\|\xi_{1 \imath}\right\|_{\infty} \\
& +\left\|\left(\hat{L}^{-1}\left(y_{p}+\zeta_{0}\right)\right)_{\imath}\right\|_{\infty}+\left\|\xi_{2 l}\right\|_{\infty} .
\end{aligned}
$$

Apparently, $\left|\xi_{1}(t)\right|$ can not grow unbounded at $t=T$ and, hence, neither can $\left|v_{p}^{(1)}(t)\right|$ due to (A.14). Obviously, $v_{p}^{(2)}$ in (A.6) can not grow unbounded either. Therefore, the result of the lemma directly follows from the contradiction.

$$
\text { Q.E.D. }
$$

Lemma A3. Consider the same problem in Lemma $A 2$ and let $e_{0}(\cdot) \in L_{x_{e}}$. Suppose the adjustable parameter vector $\theta(t)$ turns out to be uniformly bounded. Then, there exists $\mu_{1}>0$ such that for all $\mu \in\left[0, \mu_{1}\right], \hat{\xi}$ will satisfy

$$
\left\|\hat{\xi}_{t}\right\|_{\infty} \leq \alpha_{6}\left\|e_{0 t}\right\|_{\infty}+\alpha_{7} \text { for all } t \geq 0,
$$

for some $\alpha_{6}, \alpha_{7}>0$.
Proof of Lemma A3. Lemma A2 reveals that all signals belong to the extended $L_{\infty}$ space and, hence, equation (22) becomes valid for all $t \geq 0$ owing to the arguments in the beginning of Theorem 1 . Then, it can be easily seen that with the above fact (A.13) only needs to be modified slightly into the following:

$$
\left|\hat{L}^{-1}\left(v_{p}\right)\right| \leq \bar{\gamma}_{4}\left\|\hat{\xi}_{t}\right\|_{\infty}+\bar{\gamma}_{5}\left|\Delta\left(\hat{e}_{0}\right)\right|,
$$

which now remains valid for all $t \geq 0$. This, in turn, implies that (A.14) will be modified accordingly as:

$$
\begin{aligned}
\left|\dot{\xi}_{1}\right| \leq & \alpha_{3}\left\|\xi_{1 t}\right\|_{\infty}+\alpha_{4}\left\|y_{p_{t}}\right\|_{\infty} \\
& +\alpha_{5}+\bar{\alpha}_{5}\left|\Delta\left(\hat{e}_{0}\right)\right| \\
& \text { for all } t \geq 0
\end{aligned}
$$

for some $\bar{\alpha}_{5}$.

Furthermore, since $\hat{P}_{u}(s)\left(u_{p}\right)=y_{p}$, from (A.6), it is clear that

$$
\xi_{2}=\hat{P}_{u}(s)\left(\xi_{1}\right)+(s I-\Lambda)^{-1} b \hat{L}^{-1}\left(\zeta_{0}\right)+\varepsilon^{\prime}(t) . \quad \text { (A.20) }
$$

Define $\xi_{0}=(s I-\Lambda)^{-1} b \hat{L}^{-1}\left(\zeta_{0}\right)$ and recall that $\hat{P}_{u}(s)=\hat{P}$ $(s)\left(1+\mu \Delta \hat{P}_{1}(s)\right)+\mu \Delta \hat{P}_{2}(s)$. Then, it can be derived that

$$
\begin{aligned}
\xi_{3}= & : \xi_{2}-\mu \Delta \hat{P}_{2}(s)\left(\xi_{1}\right)-\xi_{0} \\
= & \hat{P}(s)\left(1+\mu \Delta \hat{P}_{1}(s)\right) \\
& \times\left(\xi_{1}\right)+\varepsilon^{\prime}(t) .
\end{aligned}
$$

By Assumption (A1) and (A3), $\hat{P}(s)$ is minimum phase with relative degree two. Now if we define $z(t)=\frac{a_{0}^{2}}{\left(s+a_{0}\right)^{2}}\left(\xi_{1}\right)$ for some $a_{0}>0$ to be determined later and borrow the results from the proof of Lemma 3.6.2 in Bodson and Sastry (1989) then we can deduce that

$$
\left\|\xi_{1 t}\right\|_{\infty} \leq\left\|z_{t}\right\|_{\infty}+\left\|\varepsilon_{f}^{\prime}\right\|_{\infty}+\frac{2}{a_{0}}\left\|\dot{\xi}_{1 s}\right\|_{\infty}
$$

and from (A.19) we can further conclude that

$$
\begin{aligned}
& \left\|\xi_{1 t}\right\|_{\infty} \leq \frac{1}{\left(1-\frac{2}{a_{0}} \alpha_{3}\right)} \\
& \times\left(\left\|z_{t}\right\|_{\infty}+\left\|\varepsilon_{f}^{\prime}\right\|_{\infty}+\frac{2}{a_{0}}\left(\alpha_{4}\left\|y_{p t}\right\|_{\infty}+\alpha_{5}+\bar{\alpha}_{5} \mid \Delta\left(\hat{e}_{0}\right)\right)\right),
\end{aligned}
$$

so long as $a_{0}$ is chosen such that $\frac{2}{a_{0}} \alpha_{3}<1$.

On the other hand, $\frac{\left(s+a_{0}\right)^{2}}{a_{0}^{2}} \hat{P}(s)$ has a stable inverse from Assumption (A3) and, hence,

$$
\begin{aligned}
z= & \frac{a_{0}^{2}}{\left(s+a_{0}\right)^{2}} \hat{P}^{-1}(s)\left(\xi_{3}-\varepsilon^{\prime}(t)\right) \\
& -\mu \Delta \hat{P}_{1}(s)(z),
\end{aligned}
$$

which implies that there exists $\mu_{0} \geq 0$ such that

$$
\left\|z_{t}\right\|_{\infty} \leq \gamma_{8}\left\|\xi_{3 t}\right\|_{\infty}+\left(\gamma_{9}+\mu \gamma_{10}\right) \text {, }
$$

for all $\mu \in\left[0, \mu_{0}\right]$ and for some appropriate positive constants $\gamma_{8}-\gamma_{10}$. It can also be shown from (A.21) that

$$
\left\|\xi_{3 t}\right\|_{\infty} \leq\left\|\xi_{2 t}\right\|_{\infty}+\mu \gamma_{11}\left\|\xi_{1 t}\right\|_{\infty}+\left(\gamma_{12}+\mu \gamma_{13}\right), \text { (A.26) }
$$

for another set of appropriate positive constants $\gamma_{11}-\gamma_{13}$. Combining (A.23), (A.25) and (A.26), we can attain the following

$$
\begin{aligned}
\left\|\xi_{1 s}\right\|_{\infty} \leq & \frac{1}{\gamma_{14}}\left(\gamma_{8}\left\|\xi_{2 s}\right\|_{\infty}+\mu\left(\gamma_{8} \gamma_{11}\right)\left\|\xi_{1 t}\right\|_{\infty}\right. \\
& \left.+\gamma_{8}\left(\gamma_{12}+\mu \gamma_{13}\right)+\left(\gamma_{9}+\mu \gamma_{12}\right)\right),
\end{aligned}
$$

where $\gamma_{14}=1-\frac{2}{a_{0}} \alpha_{3}>0$. Now, clearly, there exists $\mu_{1} \leq \mu_{0}$ such that, for all $\mu \in\left[0, \mu_{1}\right]$, (A.27) can be rewritten as:

$$
\left\|\xi_{1 /}\right\|_{\infty} \leq \gamma_{15}\left\|\xi_{2 i}\right\|_{\infty}+\gamma_{16},
$$

for some positive constants $\gamma_{15}$ and $\gamma_{16}$. Finally, it follows 
from (A.8) that

$$
\begin{aligned}
\left\|\xi_{1 t}\right\| \leq & \gamma_{2} \gamma_{15}\left\|\left(y_{p}-y_{m}\right)_{t}\right\|_{\infty} \\
& +\max _{t \geq 0}\left(\gamma_{2} \gamma_{15}\left\|y_{m t}\right\|_{\infty}+\left(\gamma_{3} \gamma_{15}+\gamma_{16}\right)\right) \\
= & \gamma_{17}\left\|e_{0 t}\right\|_{\infty}+\gamma_{18},
\end{aligned}
$$

for some $\gamma_{17}, \gamma_{18}>0$. Hence, using (A.8) and (A.29) in (A.16), we can conclude that

$$
\begin{aligned}
\left\|\hat{\xi}_{t}\right\|_{\infty} \leq & \left(\gamma_{2}+\gamma_{17}+\frac{1}{a}\right)\left\|e_{0 t}\right\|_{\infty} \\
& +\max _{t \geq 0}\left(\left(\frac{1}{a}+\gamma_{2}\right)\left\|y_{m t}\right\|_{\infty}+\left|\hat{L}^{-1}\left(r+\zeta_{0}\right)\right|\right) \\
& +\left(\gamma_{1}+\gamma_{3}+\gamma_{18}\right) \\
= & \alpha_{6}\left\|e_{0 t}\right\|_{\infty}+\alpha_{7} .
\end{aligned}
$$

This concludes out proof.

Lemma A4. Consider the same problem in Lemma A2 but let $e_{0}$ grow unbounded at some finite time $t=T<\infty$. Then, $|\xi(t)|$ will also become unbounded at $t=T$. Furthermore, if $\theta(t)$ remain uniformly bounded for all $t \in[0, T)$, then the result of Lemma $A 3$ will hold for all $t \in[0, T)$.

Proof of Lemma A4. The fact that $e_{0}$ grows unbounded at $t=T$ is equivalent to having $y_{p}$ grow unbounded at $t=T$. Recall that

$$
v_{p}^{(2)}=\frac{1}{\hat{\Lambda}(s)}\left(\begin{array}{c}
1 \\
s \\
\vdots \\
s^{n-2}
\end{array}\right)\left(y_{p}+\zeta_{0}\right),
$$

where $\hat{\Lambda}(s)$ is Hurwitz. We claim that to prove the first part of the lemma it suffices to show that $\frac{1}{\left(s+a_{0}\right)}\left(y_{p}\right)$ grows unbounded at $t=T$ for all $a_{0}>0$. The reason of the claim is given as follows: Let $\hat{\Lambda}(s)=\hat{\Lambda}_{1}(s) \hat{\Lambda}_{2}(s)$ where $\hat{\Lambda}_{1}(s)=0$ has real roots but $\hat{\Lambda}_{2}(s)=0$ only has complex roots. Moreover, let the degree of the polynomial $\hat{\Lambda}_{2}(s)$ be $n_{1}$. Then, by applying the claim successively, it is clear that the signal

$$
\frac{1}{\hat{\Lambda}_{1}(s)\left(s+a_{0}\right)^{n_{1}}}\left(y_{p}+\zeta_{0}\right)=\frac{\hat{\Lambda}_{2}(s)}{\left(s+a_{0}\right)^{n_{1}}}(x),
$$

where $x$ denotes the first element of $v_{p}^{(2)}$, becomes unbounded at $t=T$. Therefore, by use of the stable filter theory, it follows that $x$ and, hence, $\left|v_{p}^{(2)}\right|$ become unbounded at $t=T$ as well. This, in turn, says that $|\hat{w}|$ grows unbounded in finite time $t=T$ and, thus, one can deduce from the proof of Lemma $A 2$ that $|\xi|$ will also become unbounded at that same finite time. Finally, the proof of showing that $\frac{1}{\left(s+a_{0}\right)}\left(y_{p}\right)$ indeed grows unbounded at $t=T$ will be similar to that given by (A.10) in the proof of Lemma A2. This completes the first part of the proof.

To show the second part of the lemma, recall in (A.14) that

$$
\begin{gathered}
\left|\dot{\xi}_{1}\right| \leqslant \alpha_{3}\left\|\xi_{1 t}\right\|_{\infty}+\alpha_{4}\left\|y_{p t}\right\|_{\infty}+\alpha_{5} \\
\text { for all } t \in[0, T) .
\end{gathered}
$$

Consequently, it can be deduced from the proof of Lemma A3 that its result will remain valid for all $t \in[0, T)$. Q.E.D. 